\title{
Vibrational energy flow models of finite orthotropic plates
}

\author{
Do-Hyun Park ${ }^{\mathrm{a}}$, Suk-Yoon Hong ${ }^{\mathrm{a}, *}$ and Hyun-Gwon Kil ${ }^{\mathrm{b}}$ \\ ${ }^{a}$ Department of Naval Architecture and Ocean Engineering, Seoul National University, \#56-1, Shinlim-dong, \\ Kwanak-gu, Seoul 151-742, Korea \\ ${ }^{\mathrm{b}}$ Department of Mechanical Engineering, The University of Suwon, Suwon 445-743, Korea
}

\begin{abstract}
In this paper energy flow models for the transverse vibration of finite orthotropic plates are developed. These models are expressed with time- and locally space-averaged far-field energy density, and show more general forms than the conventional EFA models for isotropic plates. To verify the accuracy of the developed models, numerical analyses are performed for finite rectangular plates vibrating at a single frequency, and the calculated results expressed with the energy and intensity levels are compared with those of classical models by changing the frequency and the damping.
\end{abstract}

\section{Introduction}

The Energy Flow Analysis (EFA) method has been developed as a promising vibro-acoustic prediction tool of medium-to-high frequency ranges. The EFA method offers an improved solution to the Statistical Energy Analysis (SEA) which does not give any information on the energy variation in a subsystem and cannot consider the local power input and damping treatment. The EFA model, analogous to the steady state heat flow model, was introduced by Belov et al. [13]. Nefske and Sung [1] applied EFA to predict the flexural vibration of beams. Further studies for rods and beams were performed by Wohlever and Bernhard [3,8,9]. Bouthier and Bernhard [6-9] developed EFA models for Kirchhoff-Love plates and membranes.

Many dynamic system structures such as ships and planes include plate elements made of orthotropic materials and stiffened or reinforced plates [5,10,11]. These naturally and structurally orthotropic plates have different bending stiffnesses in two perpendicular directions, on which the energy distribution and the power transmission path depend greatly. Until now, the development of EFA models has been focused mainly

*Corresponding author. Tel.: +82 2880 8757; Fax: +82 2888 9298; E-mail: syhong@gong.snu.ac.kr. on isotropic structural elements, and more general energy flow formulations are required to apply the EFA method properly to orthotropic structures.

The aim of this work is to develop approximate energy flow models that predict the energy distribution and power transmission path in a time- and locally spaceaveraged sense for the orthotropic plates vibrating in the medium-to-high frequency ranges. Numerical analyses are performed for finite rectangular orthotropic plates which are simply supported along the edges and excited by a transverse harmonic point force. The numerical results are compared between a classical model and the derived EFA model for orthotropic plates, and the effects of frequency and damping parameters are investigated.

\section{Approximate energy flow models for the transverse vibration of orthotropic plates}

The equation of motion of thin orthotropic plates excited by a harmonic point force located at $\left(x_{o}, y_{o}\right)$ of which the amplitude and the frequency are $F$ and $\omega$, respectively, can be written as $[5,11]$ :

$$
D_{x c} \frac{\partial^{4} w}{\partial x^{4}}+2 H_{c} \frac{\partial^{4} w}{\partial x^{2} \partial y^{2}}
$$




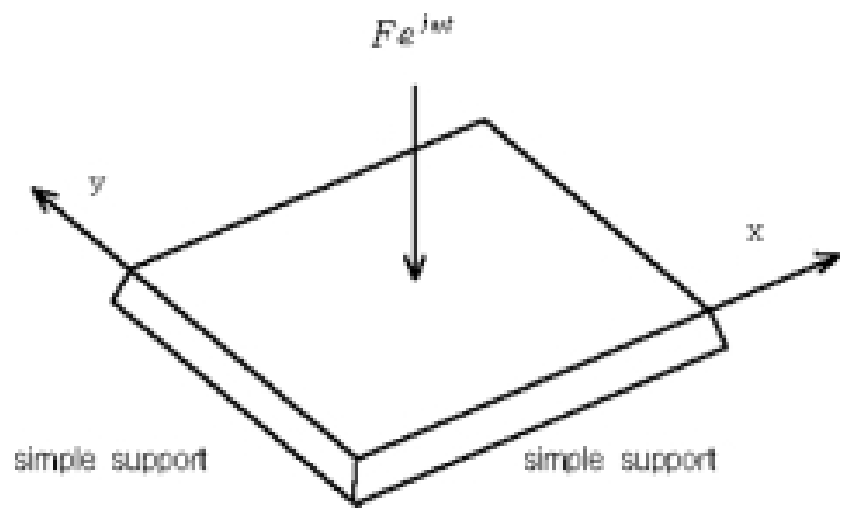

Fig. 1. The orthotropic plate response problem, displacement solution.

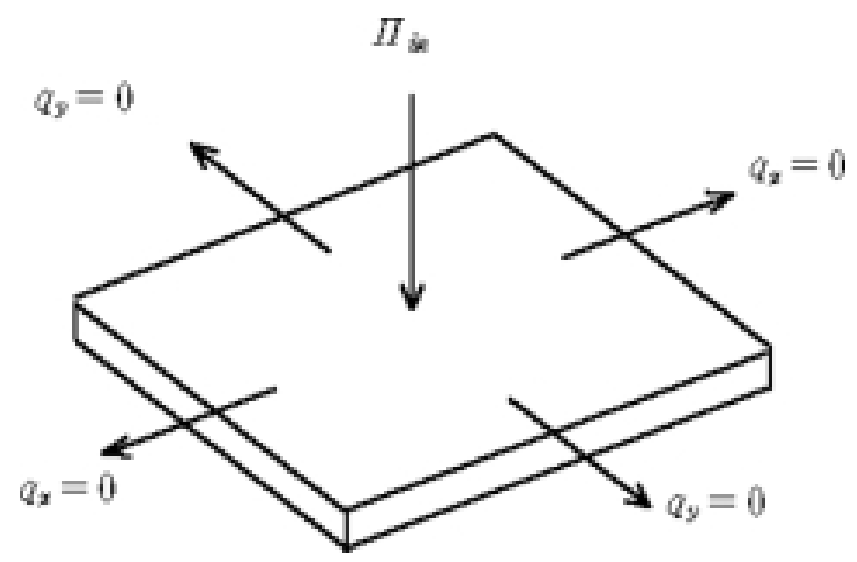

Fig. 2. The orthotropic plate response problem, energy solution.

$$
\begin{gathered}
+D_{y c} \frac{\partial^{4} w}{\partial y^{4}}+m \frac{\partial^{2} w}{\partial t^{2}} \\
=F \delta\left(x-x_{o}\right) \delta\left(y-y_{o}\right) e^{j \omega t},
\end{gathered}
$$

where $w$ is the transverse displacement and $m$ is the mass per unit area of the plate. $D_{x c}$ and $D_{y c}$ are the complex bending stiffnesses in the $x$ - and $y$-directions, respectively, and are written as

$$
\begin{aligned}
& D_{x c}=D_{x}(1+j \eta) \quad \text { and } \\
& D_{y c}=D_{y}(1+j \eta),
\end{aligned}
$$

where $\eta$ is the hysteretic damping loss factor. $H_{c}$ in Eq. (1) is the complex effective torsional stiffness. When the thickness of the plate is constant and its transverse displacement is relatively very small, its deformation being properly elastic, the effective torsional stiffness, $H_{c}$, can be assumed to be the geometric mean of the bending stiffnesses $D_{x c}$ and $D_{y c}$ in the following expression [5]:

$$
H_{c}=\sqrt{D_{x c} D_{y c}} \text {. }
$$

The effective torsional stiffness expressed by Eq. (3) makes it easy to mathematically handle the equation of motion given by Eq. (1). Despite of its preconditions, Cremer and Heckl [4] showed that Eq. (3) is a very good approximation for many practical orthotropic plates. It can be also seen in their work that the driving point impedance of an orthotropic plate is very nearly equal to that of an homogeneous plate whose bending stiffness is equal to the geometric mean of the bending stiffnesses in the two coordinate direction. Substituting Eq. (3) into Eq. (1), the equation of motion is rewritten as

$$
\begin{aligned}
& D_{x c} \frac{\partial^{2} w}{\partial x^{4}}+2 \sqrt{D_{x c} D_{y c}} \frac{\partial^{4} w}{\partial x^{2} \partial y^{2}} \\
& +D_{y c} \frac{\partial^{4} w}{\partial y^{4}}+m \frac{\partial^{2} w}{\partial t^{2}} \\
= & F \delta\left(x-x_{o}\right) \delta\left(y-y_{o}\right) e^{j \omega t} .
\end{aligned}
$$

The authors of this paper investigate approximate power flow models of the orthotropic plates with Eq. (4). 


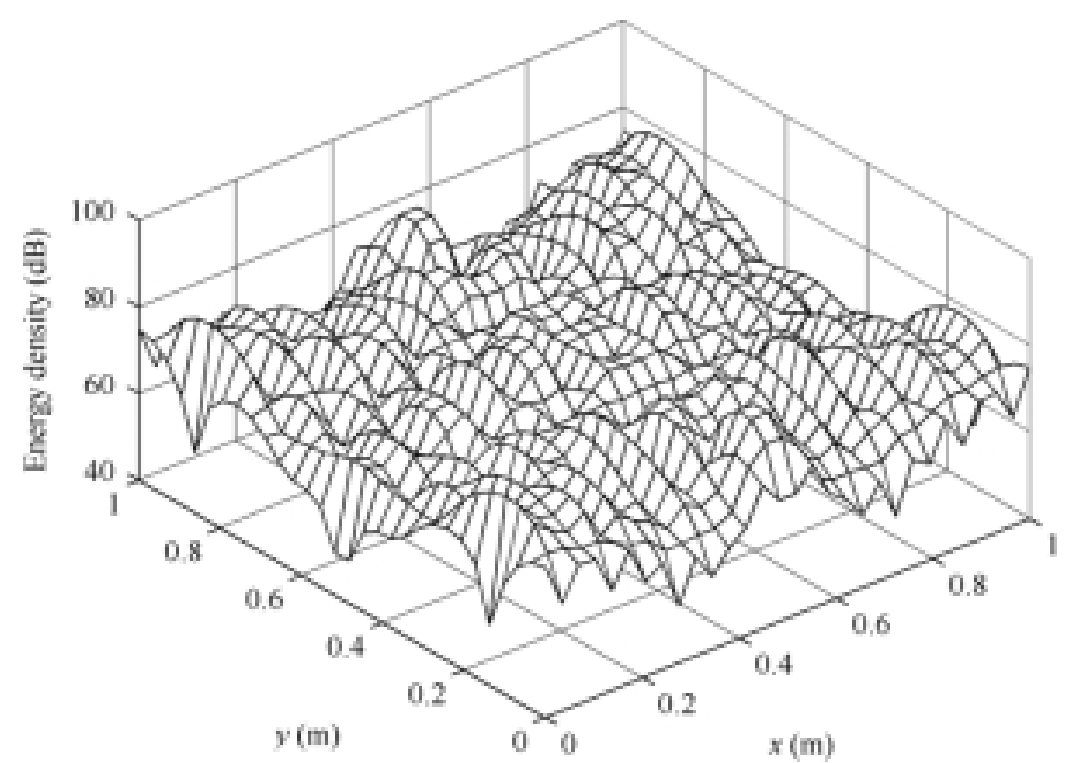

Fig. 3. The classical energy density distribution of the orthotropic plate when $f=500 \mathrm{~Hz}$ and $\eta=0.02$. The reference energy density is $1 \times 10^{-12} \mathrm{~J} / \mathrm{m}^{2}$.

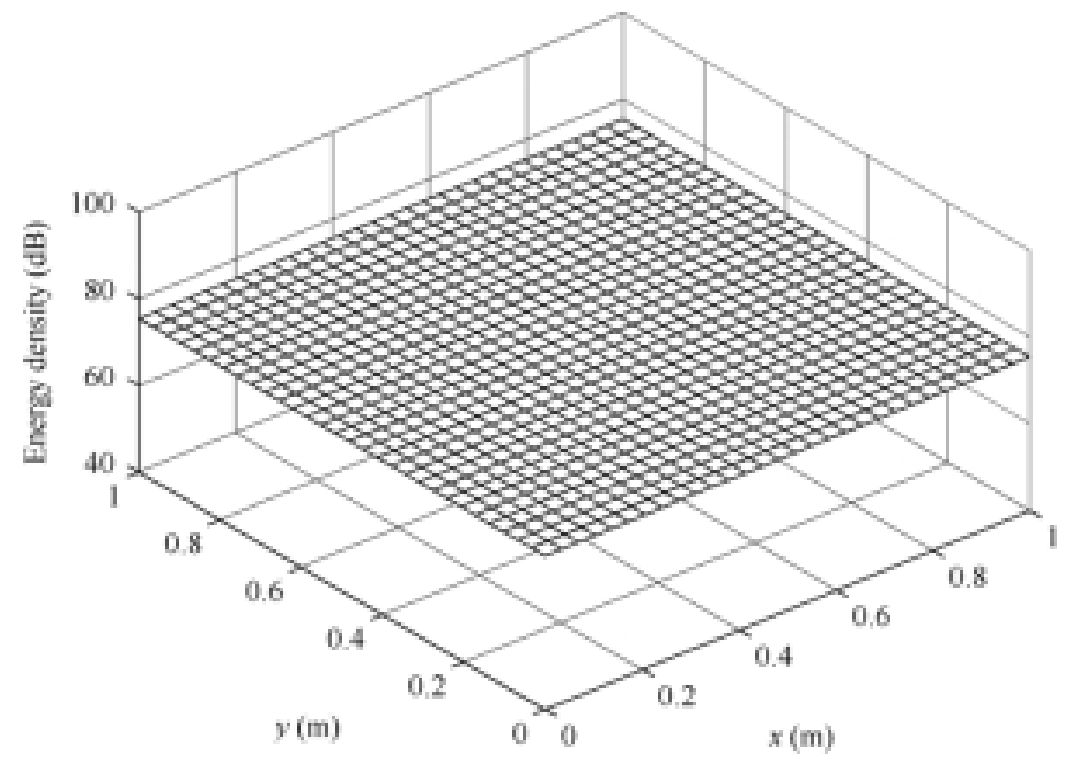

Fig. 4. The approximate energy density distribution of the orthotropic plate when $f=500 \mathrm{~Hz}$ and $\eta=0.02$. The reference energy density is $1 \times 10^{-12} \mathrm{~J} / \mathrm{m}^{2}$.

Equation (4) has both far-field and near-field solutions. The near-field waves that may be important at low frequency are commonly known to mostly affect the region within the distance of a half wavelength away from the discontinuity such as boundary and source. As the frequency increases, the near-field comes to be smaller and the far-field becomes dominant on the most area of the plate. Thus, the far-field solutions can well approximate the response at high frequency where the near-field waves may vanish quickly. It was shown by Noiseux [2] that the far-field solution is useful for the energy flow analysis of vibrating plates. The energy models for isotropic plates derived by Bouthier and Bernhard $[6,8]$ were obtained from the far-field solution of the equation of motion and appeared to be good representations of the approximate response of 


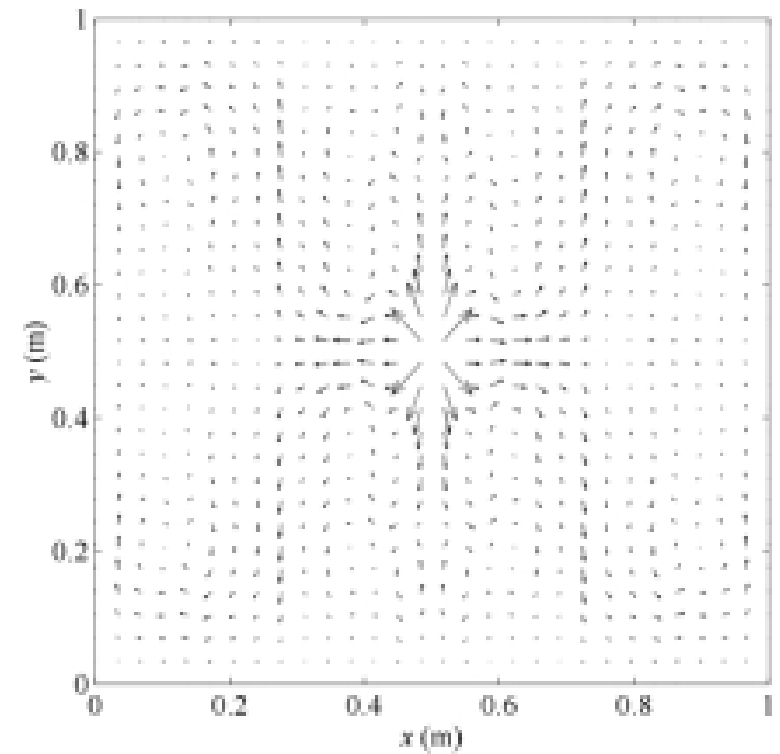

Fig. 5. The classical intensity field in the orthotropic plate when $f=500 \mathrm{~Hz}$ and $\eta=0.02$.

the plates. Thus, in this work only the far-field solutions are utilized for relevant analyses. The general far-field solution can be expressed as the sum of the plane progressive wave components:

$$
\begin{aligned}
& w_{f f}(x, y, t) \\
= & \left(A e^{-j\left(k_{x} x+k_{y} y\right)}+B e^{j\left(k_{x} x-k_{y} y\right)}\right. \\
& \left.+C e^{-j\left(k_{x} x-k_{y} y\right)}+D e^{j\left(k_{x} x+k_{y} y\right)}\right) e^{j \omega t},
\end{aligned}
$$

where $k_{x}$ and $k_{y}$ are the complex wave numbers in the $x$ - and $y$-directions, respectively. $k_{x l}$ and $k_{y l}$ are real parts of $k_{x}$ and $k_{y}$, and then the dispersion relation is expressed as

$$
\left(\sqrt{D_{x}} k_{x l}^{2}+\sqrt{D_{y}} k_{y l}^{2}\right)^{2}=\omega^{2} m
$$

When the damping is small, $k_{x}$ and $k_{y}$ are well approximated by

$$
k_{x}=k_{x l}\left(1-j \frac{\eta}{4}\right)
$$

and

$$
k_{y}=k_{y l}\left(1-j \frac{\eta}{4}\right)
$$

In general, the energy in the plates is transmitted by shear forces, bending moments and twisting moments. The shear forces $Q_{x z}$ and $Q_{y z}$ of the orthotropic plate, of which the effective torsional stiffness $H=\left(D_{x} D_{y}\right)^{1 / 2}$, are expressed by the transverse displacement as

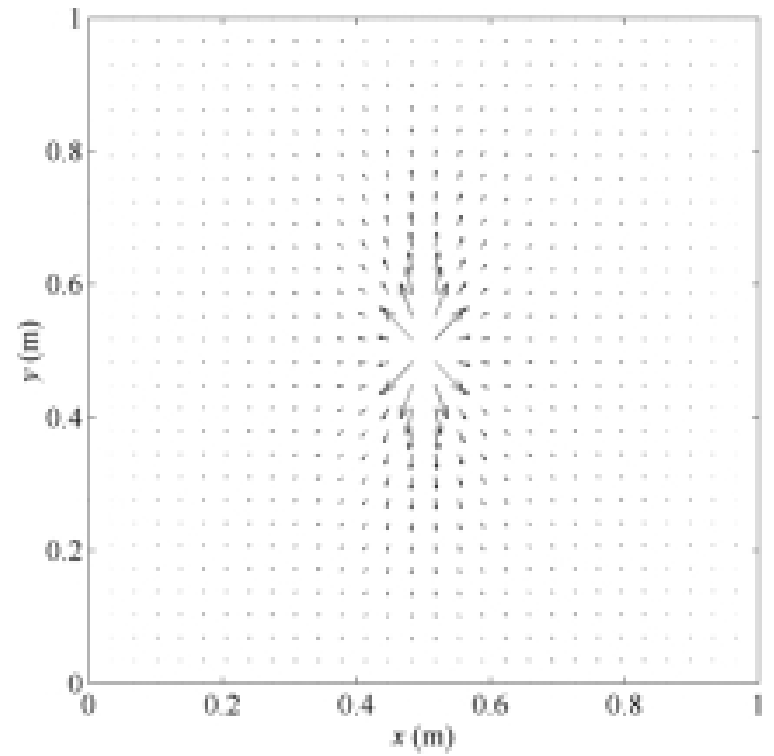

Fig. 6. The approximate intensity field in the orthotropic plate when $f=500 \mathrm{~Hz}$ and $\eta=0.02$.

$$
Q_{x z}=-\left(D_{x c} \frac{\partial^{3} w}{\partial x^{3}}+\sqrt{D_{x c} D_{y c}} \frac{\partial^{3} w}{\partial x \partial y^{2}}\right)
$$

and

$$
Q_{y z}=-\left(D_{y c} \frac{\partial^{3} w}{\partial y^{3}}+\sqrt{D_{x c} D_{y c}} \frac{\partial^{3} w}{\partial x^{2} \partial y}\right) .
$$

The bending moments, $M_{x}$ and $M_{y}$, are also written as

$$
M_{x}=-D_{x c}\left(\frac{\partial^{2} w}{\partial x^{2}}+\nu_{y} \frac{\partial^{2} w}{\partial y^{2}}\right)
$$

and

$$
M_{y}=-D_{y c}\left(\frac{\partial^{2} w}{\partial y^{2}}+\nu_{y} \frac{\partial^{2} w}{\partial x^{2}}\right)
$$

where $\nu_{x}$ and $\nu_{y}$ are the effective Poisson's ratios of the orthotropic plate in the $x$ - and $y$-directions, respectively. The twisting moment of the plates, $M_{x y}$ and $M_{y x}$, are written as

$$
M_{x y}=-\left(1-\sqrt{\nu_{x} \nu_{y}}\right) \sqrt{D_{x c} D_{y c}} \frac{\partial^{2} w}{\partial x \partial y}
$$

and

$$
M_{y x}=M_{x y} .
$$

The energy density is the linear combination of the kinetic and potential energy densities, and the timeaveraged total energy density of the orthotropic plate is 


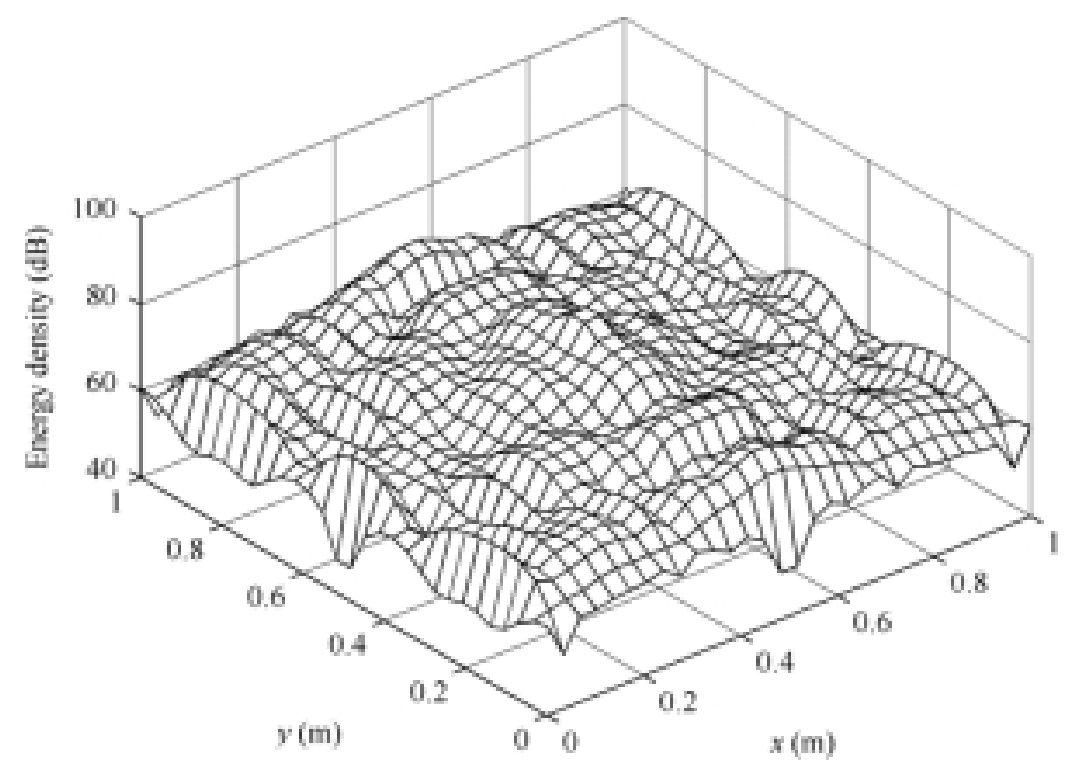

Fig. 7. The classical energy density distribution of the orthotropic plate when $f=500 \mathrm{~Hz}$ and $\eta=0.2$. The reference energy density is $1 \times 10^{-12} \mathrm{~J} / \mathrm{m}^{2}$.

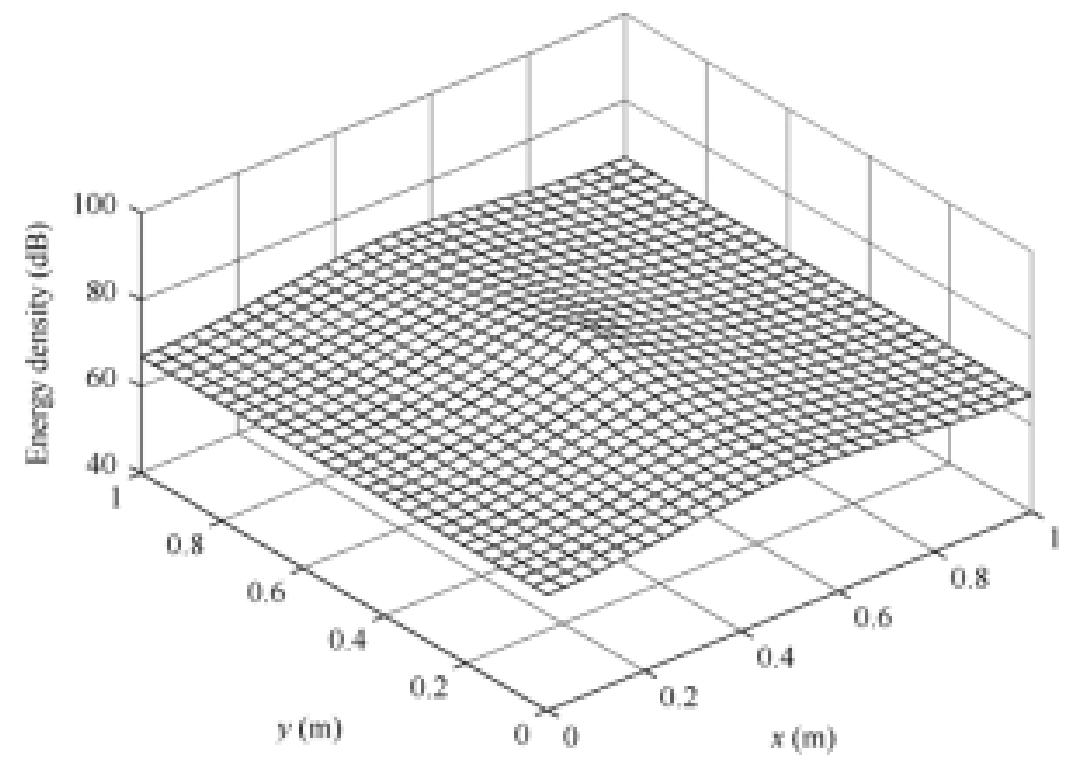

Fig. 8. The approximate energy density distribution of the orthotropic plate when $f=500 \mathrm{~Hz}$ and $\eta=0.2$. The reference energy density is $1 \times 10^{-12} \mathrm{~J} / \mathrm{m}^{2}$.

$$
\begin{aligned}
<e>= & \frac{1}{4} \operatorname{Re}\left\{D_{x c} \frac{\partial^{2} w}{\partial x^{2}}\left(\frac{\partial^{2} w}{\partial x^{2}}\right)^{*}\right. \\
& +2 \sqrt{\nu_{x} \nu_{y}} \sqrt{D_{x c} D_{y c}} \frac{\partial^{2} w}{\partial x^{2}}\left(\frac{\partial^{2} w}{\partial y^{2}}\right)^{*} \\
& +D_{y c} \frac{\partial^{2} w}{\partial y^{2}}\left(\frac{\partial^{2} w}{\partial y^{2}}\right)^{*}
\end{aligned}
$$

$$
\begin{aligned}
& +2\left(1-\sqrt{\nu_{x} \nu_{y}}\right) \sqrt{D_{x c} D_{y c}} \frac{\partial^{2} w}{\partial x \partial y} \\
& \left.\left(\frac{\partial^{2} w}{\partial x \partial y}\right)^{*}+m \frac{\partial w}{\partial t}\left(\frac{\partial w}{\partial t}\right)^{*}\right\}
\end{aligned}
$$

where $\operatorname{Re}\{\}$ represents the real part. The bracket $<>$ means the time average over a period and the superscript $*$ indicates the complex conjugate. The $x$ 


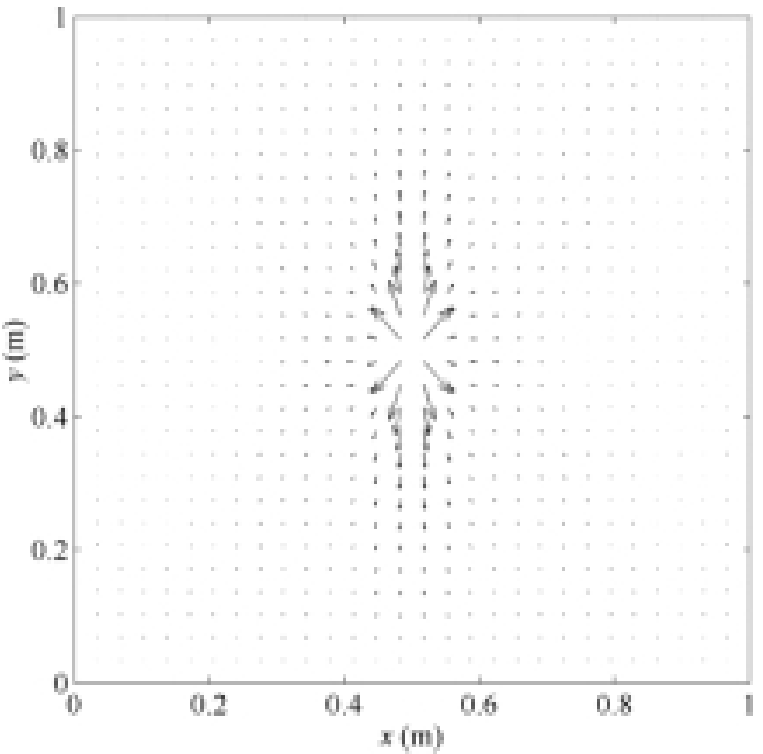

Fig. 9. The classical intensity field in the orthotropic plate when $f=500 \mathrm{~Hz}$ and $\eta=0.2$.

and $y$ components of the time-averaged intensity of a vibrating orthotropic plate are expressed by the shear forces, bending moments, twisting moments and the transverse displacement as follows:

$$
\begin{aligned}
<q_{x}>= & \frac{1}{2} \operatorname{Re}\left\{-Q_{x z}\left(\frac{\partial w}{\partial t}\right)^{*}\right. \\
& +M_{x}\left(\frac{\partial^{2} w}{\partial x \partial t}\right)^{*} \\
& \left.+M_{x y}\left(\frac{\partial^{2} w}{\partial y \partial t}\right)^{*}\right\}
\end{aligned}
$$

and

$$
\begin{aligned}
<q_{y}>= & \frac{1}{2} \operatorname{Re}\left\{-Q_{y z}\left(\frac{\partial w}{\partial t}\right)^{*}\right. \\
& +M_{y}\left(\frac{\partial^{2} w}{\partial y \partial t}\right)^{*} \\
& \left.+M_{y x}\left(\frac{\partial^{2} w}{\partial x \partial t}\right)^{*}\right\}
\end{aligned}
$$

The far-field energy density can be obtained by substituting Eq. (5) into Eq. (11). The expression for the time-averaged far-field energy density expanded in terms of the constants $A, \ldots, D$ in Eq. (5) becomes

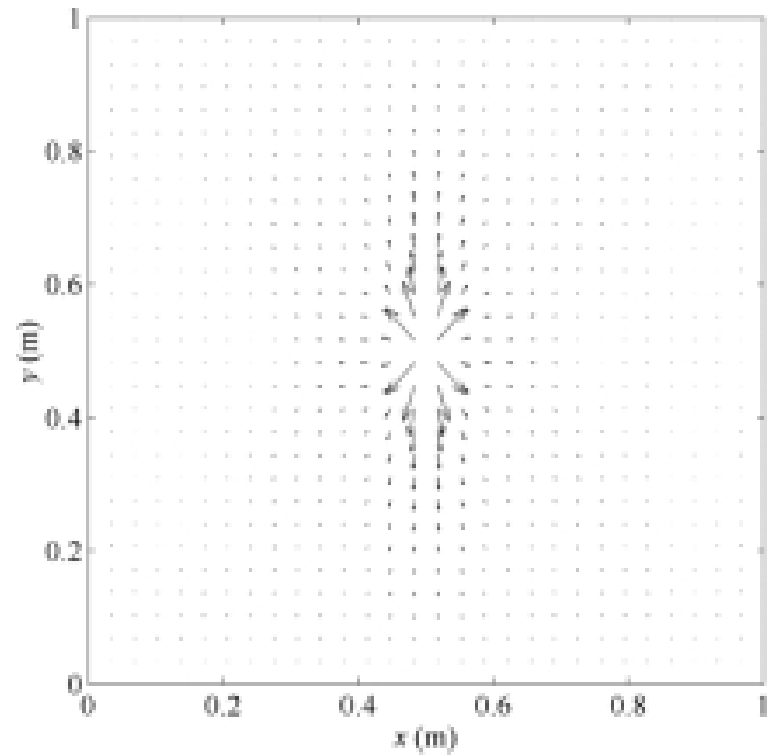

Fig. 10. The approximate intensity field in the orthotropic plate when $f=500 \mathrm{~Hz}$ and $\eta=0.2$.

$$
\begin{aligned}
& <e>= \\
& \frac{1}{4} \operatorname{Re}\left\{D_{x c}\left|k_{x}\right|^{4}[A]^{--}\right. \\
& +[B]^{+-}+[C]^{-+}+\left.[D]^{++}\right|^{2} \\
& +2 \sqrt{\nu_{x} \nu_{y}} \sqrt{D_{x c} D_{y c}} k_{x}^{2}\left(k_{y}^{2}\right)^{*} \\
& \left|[A]^{--}+[B]^{+-}+[C]^{-+}+[D]^{++}\right|^{2} \\
& +2\left(1-\sqrt{\nu_{x} \nu_{y}}\right) \sqrt{D_{x c} D_{y c}}\left|k_{x}\right|^{2}\left|k_{y}\right|^{2} \\
& \left|[A]^{--}-[B]^{+-}-[C]^{-+}+[D]^{++}\right|^{2} \\
& +D_{y c}\left|k_{y}\right|^{4}\left|[A]^{--}+[B]^{+-}+[C]^{-+}+[D]^{++}\right|^{2} \\
& \left.+m \omega^{2}\left|[A]^{--}+[B]^{+-}+[C]^{-+}+[D]^{++}\right|^{2}\right\}
\end{aligned}
$$

where []$^{ \pm \pm}$means []$\times \exp \left( \pm j k_{x} x \pm j k_{y} y\right)$. The $x$ and $y$ components of the far-field intensity can be obtained by substituting Eq. (5) into Eq. (12). The expanded expressions for the time-averaged far-field intensity are written as

$$
\begin{aligned}
& <q_{x}> \\
= & \frac{\omega}{2} \operatorname{Re}\left\{k_{x}\left(D_{x c} k_{x}^{2}+\sqrt{D_{x c} D_{y c}} k_{y}^{2}\right)\right. \\
& \times\left([A]^{--}-[B]^{+-}+[C]^{-+}-[D]^{++}\right) \\
& \left([A]^{--}+[B]^{+-}+[C]^{-+}+[D]^{++}\right)^{*} \\
& +D_{x c}\left(k_{x}^{2}+\nu_{y} k_{y}^{2}\right) k_{x}^{*} \\
& \times\left([A]^{--}+[B]^{+-}+[C]^{-+}+[D]^{++}\right) \\
& \left([A]^{--}-[B]^{+-}+[C]^{-+}-[D]^{++}\right)^{*} \\
& +\left(1-\sqrt{\nu_{x} \nu_{y}}\right) \sqrt{D_{x c} D_{y c}} k_{x}\left|k_{y}\right|^{2}
\end{aligned}
$$




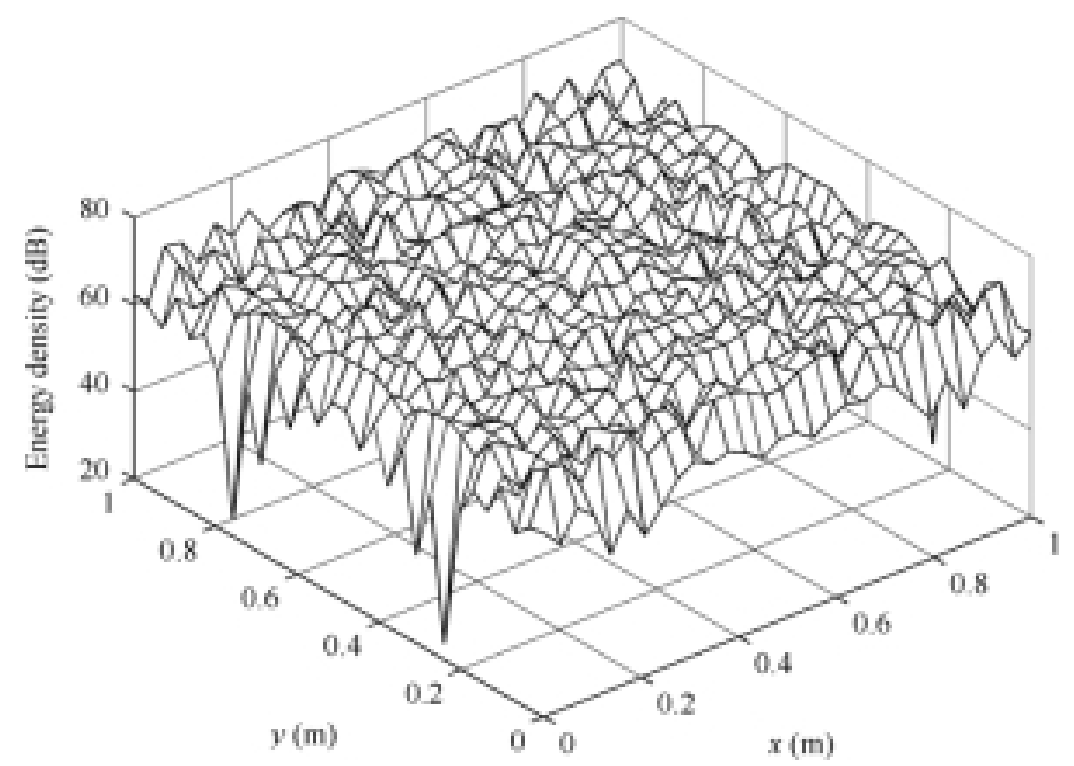

Fig. 11. The classical energy density distribution of the orthotropic plate when $f=5 \mathrm{kHz}$ and $\eta=0.02$. The reference energy density is $1 \times 10^{-12} \mathrm{~J} / \mathrm{m}^{2}$.

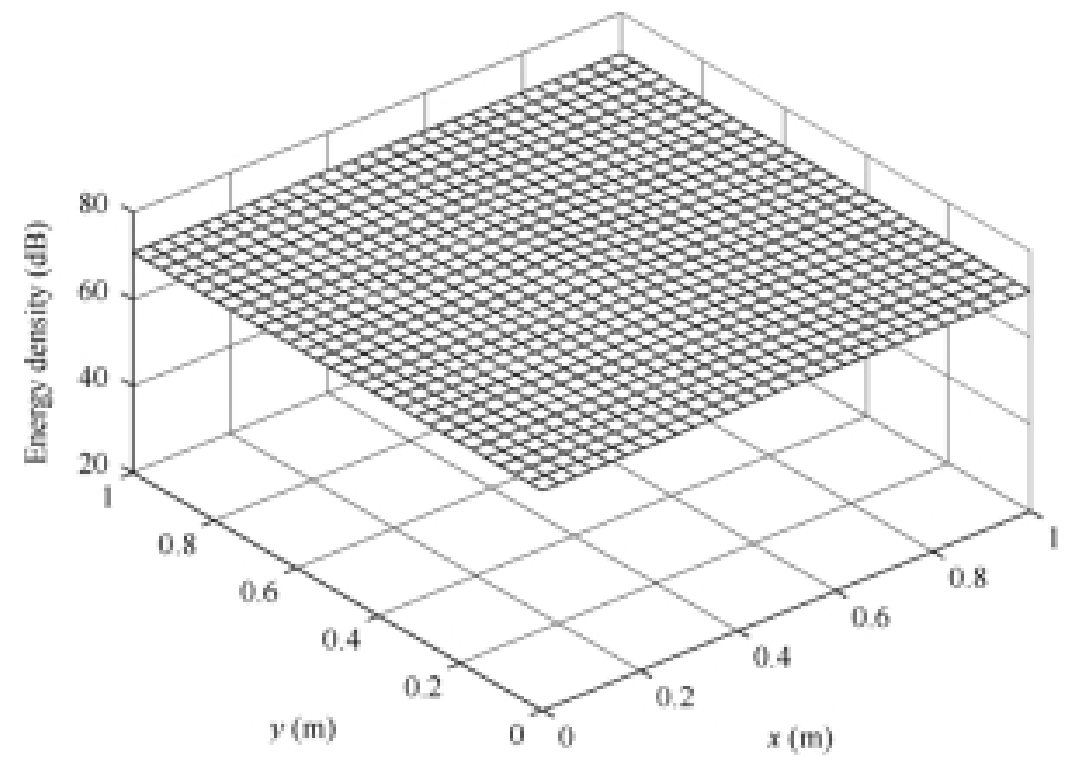

Fig. 12. The approximate energy density distribution of the orthotropic plate when $f=5 \mathrm{kHz}$ and $\eta=0.02$. The reference energy density is $1 \times 10^{-12} \mathrm{~J} / \mathrm{m}^{2}$.

$$
\begin{aligned}
& \times\left([A]^{--}-[B]^{+-}-[C]^{-+}+[D]^{++}\right) \\
& \left([A]^{--}+[B]^{+-}-[C]^{-+}-[D]^{++}\right)^{*}
\end{aligned}
$$

and

$$
\begin{aligned}
& <q_{y}> \\
= & \frac{\omega}{2} \operatorname{Re}\left\{k_{y}\left(D_{y c} k_{y}^{2}+\sqrt{D_{x c} D_{y c}} k_{x}^{2}\right)\right.
\end{aligned}
$$

$$
\begin{aligned}
& \times\left([A]^{--}+[B]^{+-}-[C]^{-+}-[D]^{++}\right) \\
& \left([A]^{--}+[B]^{+-}+[C]^{-+}+[D]^{++}\right)^{*} \\
& +D_{y c}\left(k_{y}^{2}+\nu_{y} k_{y}^{2}\right) k_{y}^{*} \\
& \times\left([A]^{--}+[B]^{+-}+[C]^{-+}+[D]^{++}\right) \\
& \left([A]^{--}+[B]^{+-}-[C]^{-+}-[D]^{++}\right)^{*} \\
& +\left(1-\sqrt{\nu_{x} \nu_{y}}\right) \sqrt{D_{x c} D_{y c}}\left|k_{x}\right|^{2} k_{y}
\end{aligned}
$$




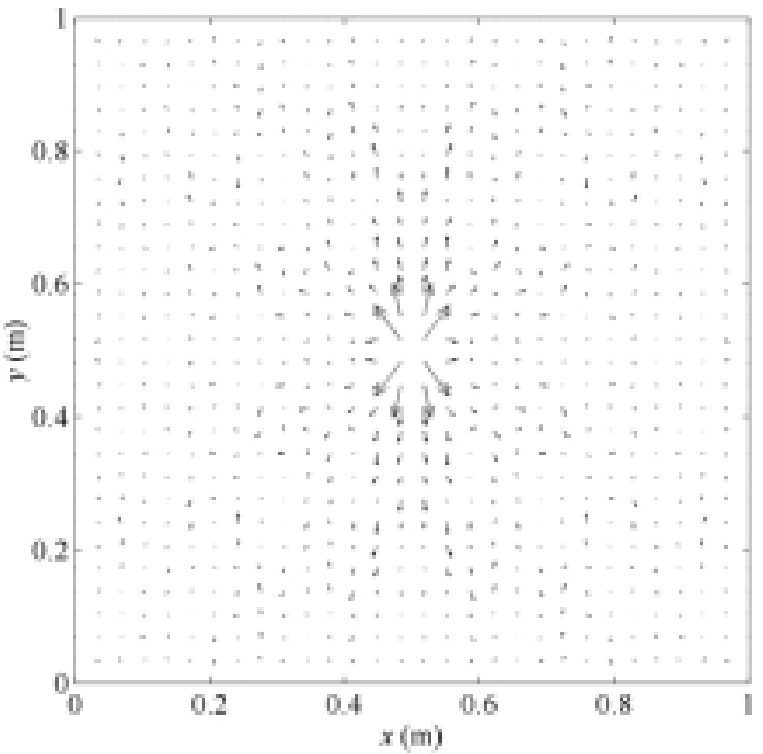

Fig. 13. The classical intensity field in the orthotropic plate when $f=5 \mathrm{kHz}$ and $\eta=0.02$.

$$
\begin{aligned}
& \times\left([A]^{--}-[B]^{+-}-[C]^{-+}+[D]^{++}\right) \\
& \left([A]^{--}-[B]^{+-}+[C]^{-+}-[D]^{++}\right)^{*}
\end{aligned}
$$

Equations (13), (14) and (15) consist of purely exponentially decayed terms $\left(\left|[A]^{--}\right|^{2}, \ldots,\left|[D]^{++}\right|^{2}\right)$ and spatially harmonic terms $\left([A]^{--}\left([B]^{+-}\right)^{*},[C]^{-+}\right.$ $\left([B]^{+-}\right)^{*}$, etc. $)$. At this stage, no obvious relations between the energy density (Eq. (13)) and the intensity (Eqs (14) and (15)) are found. Thus, the time-averaged far-field energy density and intensity are spatially averaged over a half wavelength for small damping in the following manner $[6,8]$ :

$$
\begin{aligned}
<\tilde{e}>= & \frac{k_{x l} k_{y l}}{\pi^{2}} \int_{0}^{\pi / k_{y l}} \int_{0}^{\pi / k_{x l}} \\
& <e>d x d y
\end{aligned}
$$

and

$$
\begin{aligned}
<\tilde{\mathbf{q}}>= & \frac{k_{x l} k_{y l}}{\pi^{2}} \int_{0}^{\pi / k_{y l}} \int_{0}^{\pi / k_{x l}} \\
& <\tilde{\mathbf{q}}>d x d y,
\end{aligned}
$$

where $<\tilde{e}>$ and $<\tilde{\mathbf{q}}>$ are the time- and spaceaveraged energy density and intensity, respectively. Neglecting all of the second and higher order terms of the damping loss factor yields the simplified expression for the energy density as follows (Appendix 1):

$$
\begin{aligned}
<\tilde{e}>= & \frac{1}{2} \omega^{2} m\left(|A|^{2} e^{--}+|B|^{2} e^{+-}\right. \\
& \left.+|C|^{2} e^{-+}+|D|^{2} e^{++}\right),
\end{aligned}
$$

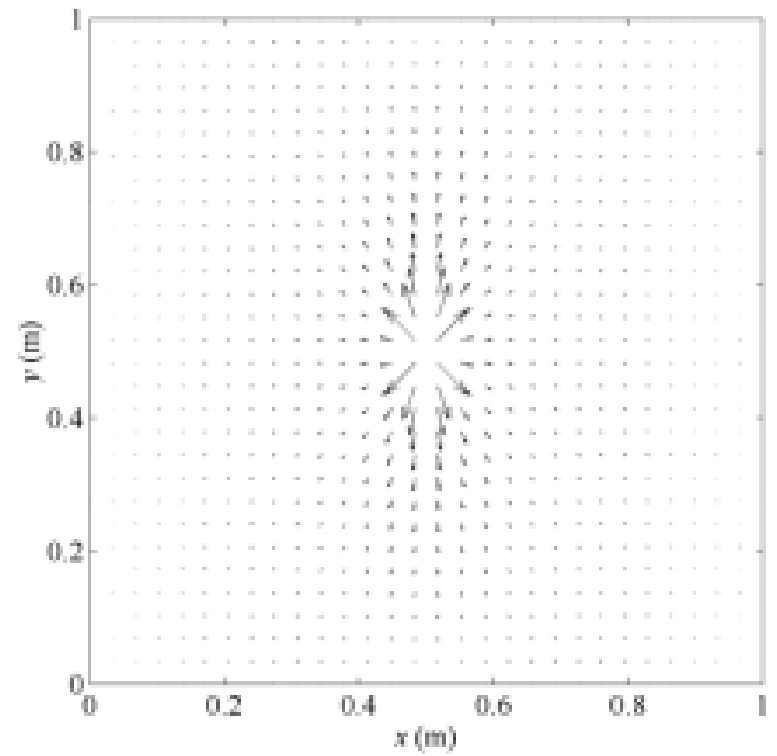

Fig. 14. The approximate intensity field in the orthotropic plate when $f=5 \mathrm{kHz}$ and $\eta=0.02$.

where $e^{ \pm \pm}$means $\exp \left\{ \pm(\eta / 2) k_{x l} x \pm(\eta / 2) k_{y l} y\right\}$. The total energy density is represented as the sum of the energy densities of four plane wave components. The simplified expressions for the $x$ and $y$ components of the time- and space-averaged intensity are written as

$$
\begin{aligned}
<\tilde{q}_{x}>= & \omega k_{x l} \sqrt{D_{x}} \sqrt{\omega^{2} m} \\
& \left(|A|^{2} e^{--}-|B|^{2} e^{+-}\right. \\
& \left.+|C|^{2} e^{-+}-|D|^{2} e^{++}\right),
\end{aligned}
$$

and

$$
\begin{aligned}
<\tilde{q}_{y}>= & \omega k_{y l} \sqrt{D_{y}} \sqrt{\omega^{2} m} \\
& \left(|A|^{2} e^{--}+|B|^{2} e^{+-}\right. \\
& \left.-|C|^{2} e^{-+}+|D|^{2} e^{++}\right),
\end{aligned}
$$

The net intensity is represented as the subtraction of the intensities of the waves propagating in the negative direction from those of the waves propagating in the positive direction. From Eqs (18), (19) and (20) and the dispersion relation (Eq. (6)), we find that the $x$ and $y$ components of intensity are proportional to the first derivatives of the energy density with respect to $x$ and $y$, respectively as

$$
<\tilde{q}_{x}>=-\frac{4}{\eta \omega} \sqrt{\frac{\omega^{2} D_{x}}{m}} \frac{\partial<\tilde{e}>}{\partial x}
$$

and

$$
<\tilde{q}_{y}>=-\frac{4}{\eta \omega} \sqrt{\frac{\omega^{2} D_{y}}{m}} \frac{\partial<\tilde{e}>}{\partial y} .
$$




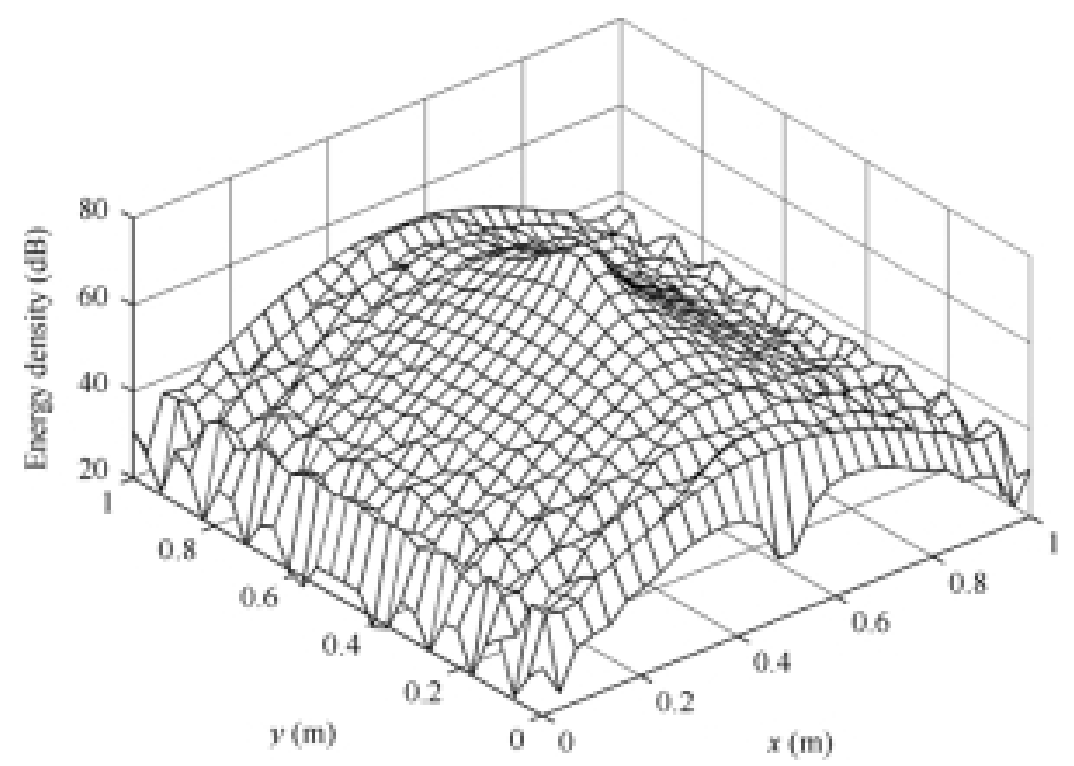

Fig. 15. The classical energy density distribution of the orthotropic plate when $f=5 \mathrm{kHz}$ and $\eta=0.2$. The reference energy density is $1 \times 10^{-12} \mathrm{~J} / \mathrm{m}^{2}$.

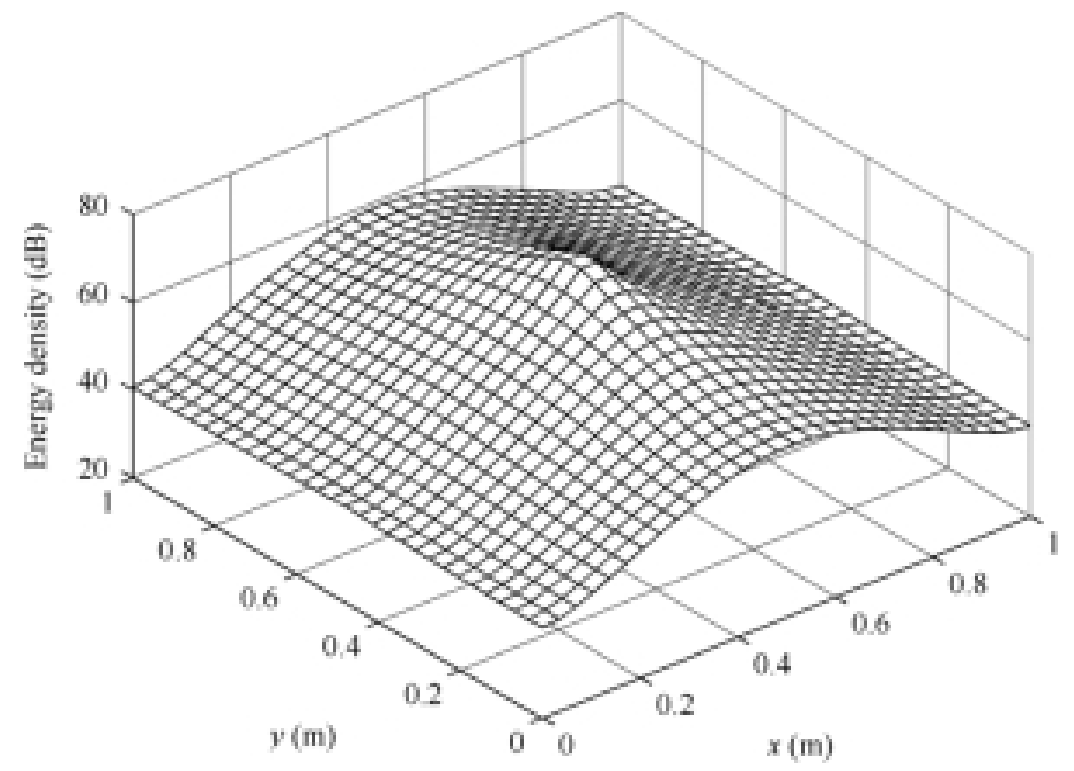

Fig. 16. The approximate energy density distribution of the orthotropic plate when $f=5 \mathrm{kHz}$ and $\eta=0.2$. The reference energy density is $1 \times 10^{-12} \mathrm{~J} / \mathrm{m}^{2}$.

Consequently, by rearranging Eq. (21) into a vector form, the intensity can be rewritten as

$$
<\tilde{\mathbf{q}}>=-\left(\frac{c_{g x}^{2}}{\eta \omega} \frac{\partial}{\partial x} \mathbf{i}+\frac{c_{g y}^{2}}{\eta \omega} \frac{\partial}{\partial y} \mathbf{j}\right)<\tilde{e}>,
$$

where $c_{g x}$ and $c_{g y}$ are defined in the following expressions:

$$
c_{g x}=2\left(\frac{\omega^{2} D_{x}}{m}\right)^{1 / 4}
$$

and

$$
c_{g y}=2\left(\frac{\omega^{2} D_{y}}{m}\right)^{1 / 4} .
$$

Equation (22) is the expression for the vibrational energy transmission in an orthotropic plate, where it 


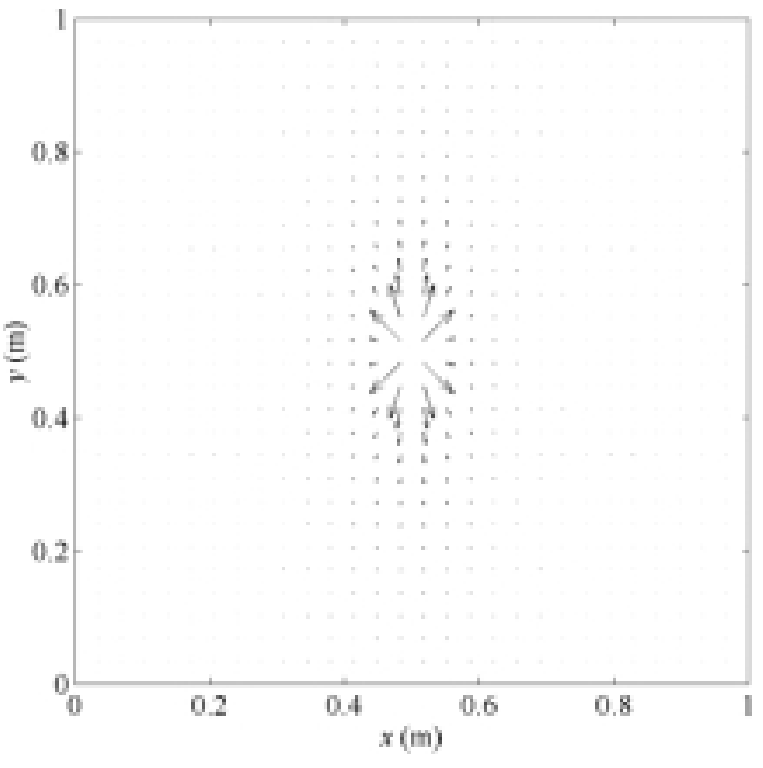

Fig. 17. The classical intensity field in the orthotropic plate when $f=5 \mathrm{kHz}$ and $\eta=0.2$.

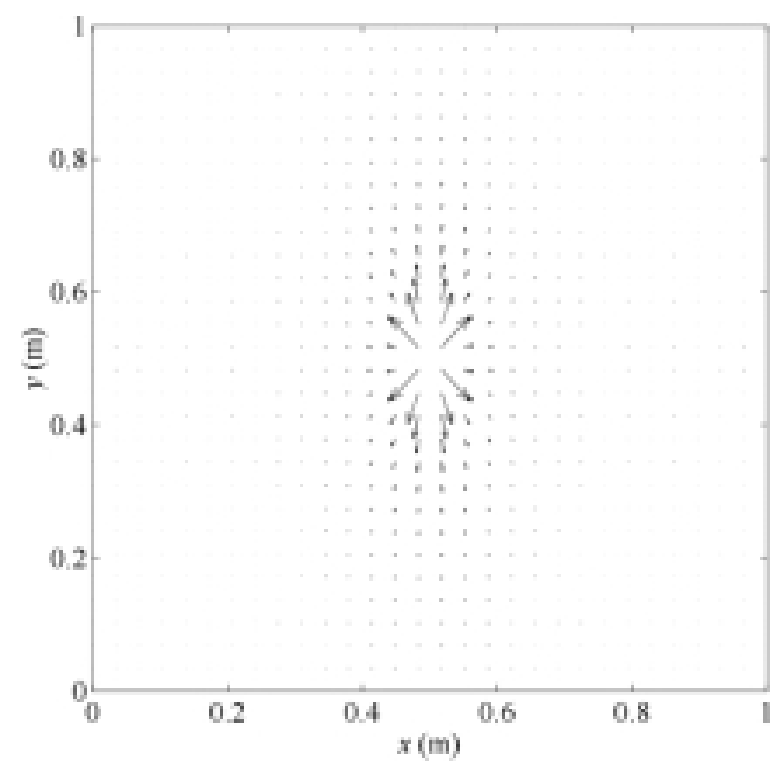

Fig. 18. The approximate intensity field in the orthotropic plate when $f=5 \mathrm{kHz}$ and $\eta=0.2$.

can be found that the intensity of orthotropic plate is not proportional to the gradient of the energy density since the bending stiffness can be different in two perpendicular directions.

The power injected by external loads into an elastic medium is dissipated due to damping and is transmitted to the next media. For the steady state elastic system, the power balance equation can be written as

$$
\nabla \cdot \mathbf{q}+\pi_{\text {diss }}=\pi_{\text {in }},
$$

where $\pi_{\text {diss }}$ and $\pi_{\text {in }}$ are the dissipated power due to the damping of the system and the input power, respectively. From the work of Cremer and Heckl [4], the time-averaged dissipated power in an elastic medium with small structural damping $(\eta \ll 1)$ is proportional to the time-averaged total energy density in the form:

$$
<\pi_{\text {diss }}>=\eta \omega<e>
$$

where it is assumed that the kinetic and potential energies of the medium are approximately the same. Combination of the power balance Eq. (Eq. (24)) with the energy transmission relation (Eq. (22)) and the power dissipation relation (Eq. (25)) yields the second order partial differential equation that takes the total energy density as a primary variable:

$$
\begin{aligned}
& -\left(\frac{c_{g x}^{2}}{\eta \omega} \frac{\partial^{2}}{\partial x^{2}}+\frac{c_{g y}^{2}}{\eta \omega} \frac{\partial^{2}}{\partial y^{2}}\right)<\tilde{e}> \\
& +\eta \omega<\tilde{e}>=<\tilde{\pi}_{\text {in }}>.
\end{aligned}
$$

Equation (26) is the differential equation for the timeand space-averaged far-field energy density of a vibrating orthotropic plate. If the bending stiffnesses $D_{x}$ and $D_{y}$ of the plate are equal to $D, c_{g x}$ and $c_{g y}$ then become

$$
c_{g x}=c_{g y}=c_{g}=2\left(\frac{\omega^{2} D}{m}\right)^{1 / 4},
$$

where $c_{g}$ is the group velocity of the corresponding isotropic plate. In this specific case, the energy governing equation for the isotropic plate is deduced from Eq. (26) as

$$
\begin{aligned}
& -\frac{c_{g x}^{2}}{\eta \omega}\left(\frac{\partial^{2}}{\partial x^{2}}+\frac{\partial^{2}}{\partial y^{2}}\right)<\tilde{e}> \\
& +\eta \omega<\tilde{e}>=<\tilde{\pi}_{\text {in }}>
\end{aligned}
$$

which is the same equation developed by Bouthier and Bernhard.

\section{Energy flow analysis of a rectangular orthotropic plate}

\subsection{Analytical solution}

In this section, the developed energy flow model is applied to the rectangular orthotropic plate simply supported along its edges and excited by a transverse point force at a single frequency. When the force is located at $\left(x_{o}, y_{o}\right)$ of the plate, the energy Eq. (26) 


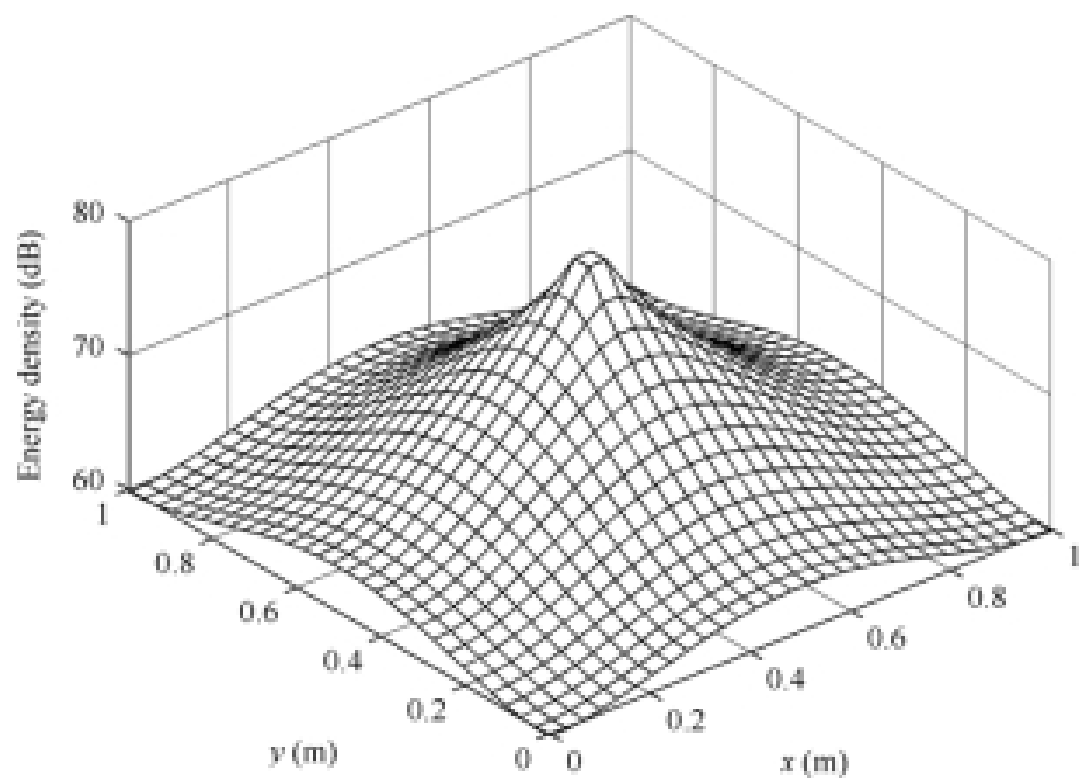

Fig. 19. The approximate energy density distribution of the isotropic plate when $f=1 \mathrm{kHz}$ and $\eta=0.2$. The reference energy density is $1 \times 10^{-12} \mathrm{~J} / \mathrm{m}^{2}$.

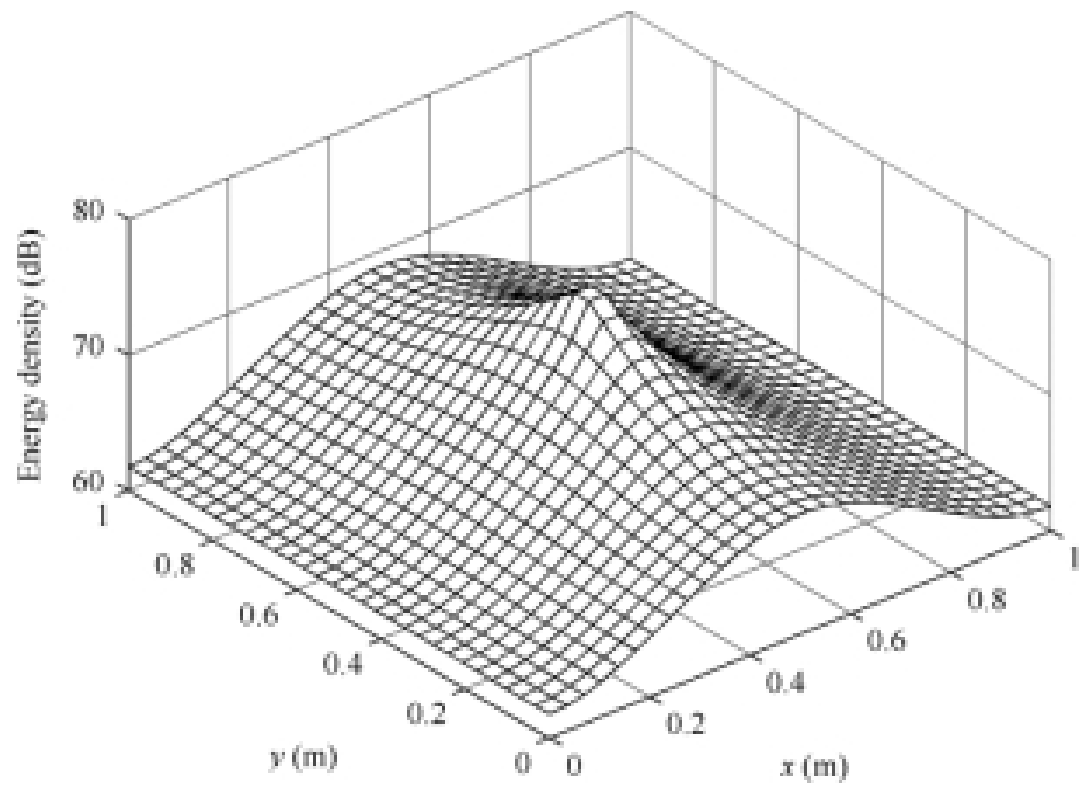

Fig. 20. The approximate energy density distribution of the orthotropic plate when $f=1 \mathrm{kHz}$ and $\eta=0.2$. The reference energy density is $1 \times 10^{-12} \mathrm{~J} / \mathrm{m}^{2}$.

becomes

$$
\begin{aligned}
& -\left(\frac{c_{g x}^{2}}{\eta \omega} \frac{\partial^{2}}{\partial x^{2}}+\frac{c_{g y}^{2}}{\eta \omega} \frac{\partial^{2}}{\partial y^{2}}\right)<\tilde{e}> \\
& +\eta \omega<\tilde{e}>=\prod_{\text {in }} \delta\left(x-x_{o}\right) \delta\left(y-y_{o}\right),
\end{aligned}
$$

where $\prod_{\text {in }}$ is the time-averaged power injected by the exciting force. When the plate is simply supported, free or fixed at its boundary, it can be assumed that there is no outflow of the power from the boundary, and thus the energy density solution of Eq. (29) can be expressed by the double Fourier series of the cosine functions with respect to the spatial variables $x$ and $y[9,12]$ : 


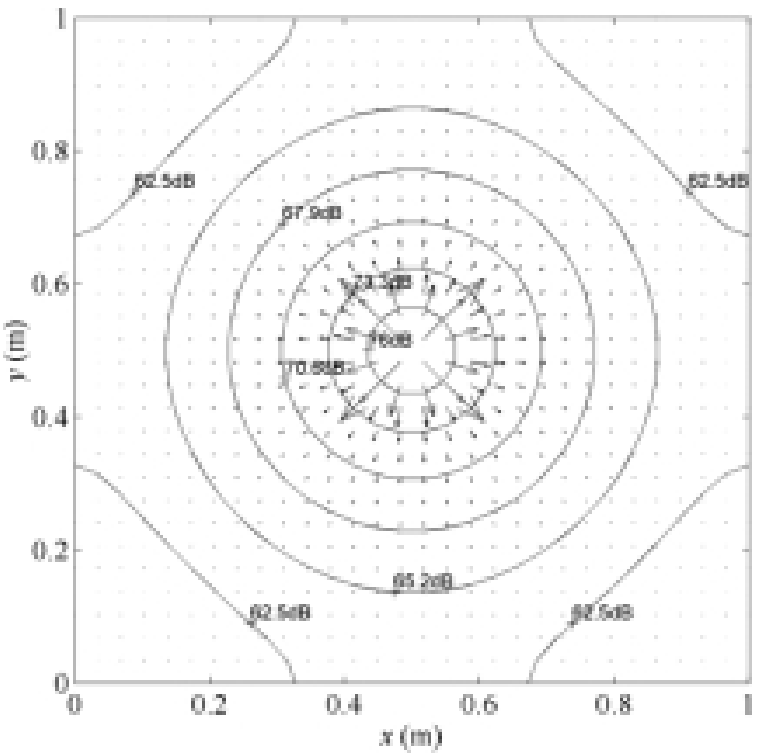

Fig. 21. The approximate intensity field with the contour plot of energy density in the isotropic plate when $f=1 \mathrm{kHz}$ and $\eta=0.2$. The reference energy density is $1 \times 10^{-12} \mathrm{~J} / \mathrm{m}^{2}$.

$$
\begin{aligned}
<\tilde{e}>= & \sum_{m=0}^{\infty} \sum_{n=0}^{\infty} E_{m n} \cos \left(\frac{m \pi}{L_{x}} x\right) \\
& \cos \left(\frac{n \pi}{L_{y}} y\right),
\end{aligned}
$$

where $E_{m n}$ is the $(m, n)$ mode coefficient of the energy density solution, and $L_{x}$ and $L_{y}$ are the dimensions of the plate. The input power can also be represented in the form of the cosine series:

$$
\begin{aligned}
& \prod_{\mathrm{in}} \delta\left(x-x_{o}\right) \delta\left(y-y_{o}\right) \\
= & \sum_{m=0}^{\infty} \sum_{n=0}^{\infty} \prod_{m n} \cos \left(\frac{m \pi}{L_{x}} x\right) \cos \left(\frac{n \pi}{L_{y}} y\right),
\end{aligned}
$$

where the coefficient of the $(m, n)$ mode, $\prod_{m n}$, is

$$
\begin{aligned}
\prod_{m n}= & \frac{A_{m n}}{L_{x} L_{y}} \prod_{i n} \cos \left(\frac{m \pi}{L_{x}} x_{o}\right) \\
& \cos \left(\frac{n \pi}{L_{y}} y_{o}\right) .
\end{aligned}
$$

Here, the factor $A_{m n}$ is determined by

$$
A_{m n}=\left\{\begin{aligned}
1 & : n=0 \text { and } n=0 \\
2 & :(m=0 \text { and } n \neq 0) \text { or } \\
& (m \neq 0 \text { and } n=0) . \\
4: & m \neq 0 \text { and } n \neq 0
\end{aligned}\right.
$$

Substituting Eqs (30) and (31) into the energy differential Eq. (29) yields the modal coefficient of the

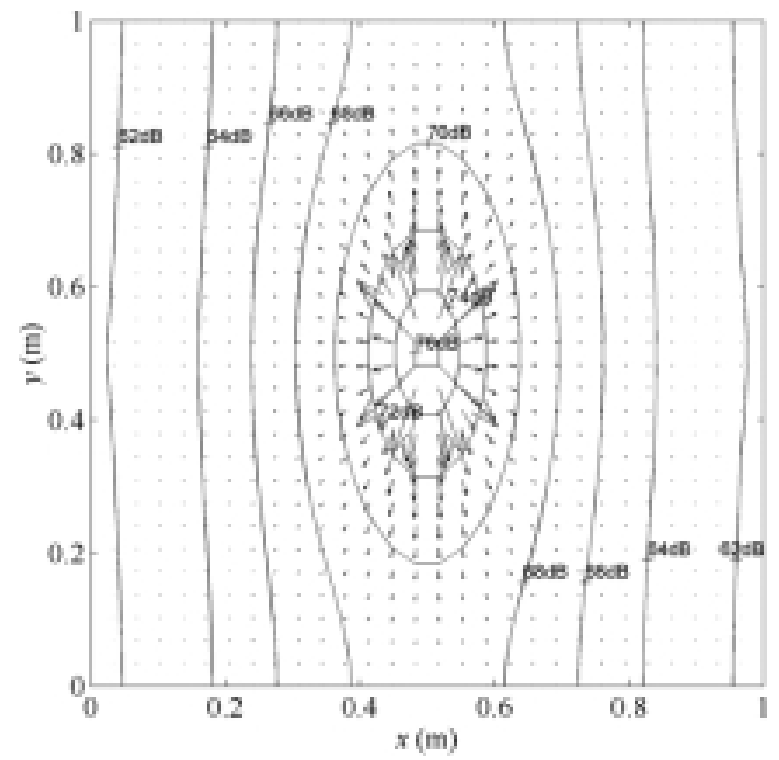

Fig. 22. The approximate intensity field with the contour plot of energy density in the orthotropic plate when $f=1 \mathrm{kHz}$ and $\eta=0.2$. The reference energy density is $1 \times 10^{-12} \mathrm{~J} / \mathrm{m}^{2}$.

energy density solution in the following expression:

$$
\begin{aligned}
& E_{m n} \\
= & \frac{\frac{A_{m n}}{L_{x} L_{y}} \prod_{\text {in }} \cos \left(\frac{m \pi}{L_{x}} x_{o}\right) \cos \left(\frac{n \pi}{L_{y}} y_{o}\right)}{\frac{c_{g x}^{2}}{\eta \omega}\left(\frac{m \pi}{L_{x}}\right)^{2}+\frac{c_{g y}}{\eta \omega}\left(\frac{n \pi}{L_{y}}\right)^{2}+\eta \omega} .
\end{aligned}
$$

The $x$ and $y$ components of the intensity can be obtained by substituting the energy density solution expressed by Eq. (30) into the energy transmission relation represented by Eq. (21) or Eq. (22):

$$
\begin{aligned}
<\tilde{q}_{x}>= & \frac{c_{g x}^{2}}{\eta \omega} \sum_{m=1}^{\infty} \sum_{n=0}^{\infty} E_{m n}\left(\frac{m \pi}{L_{x}}\right) \\
& \sin \left(\frac{m \pi}{L_{x}} x\right) \cos \left(\frac{n \pi}{L_{y}} y\right)
\end{aligned}
$$

and

$$
\begin{aligned}
<\tilde{q}_{y}>= & \frac{c_{g y}^{2}}{\eta \omega} \sum_{m=0}^{\infty} \sum_{n=1}^{\infty} E_{m n}\left(\frac{n \pi}{L_{y}}\right) \\
& \cos \left(\frac{m \pi}{L_{x}} x\right) \sin \left(\frac{n \pi}{L_{y}} y\right) .
\end{aligned}
$$

\subsection{Numerical examples}

Numerical analyses are performed for the finite rectangular orthotropic plate simply supported along its edges and excited by a transverse harmonic point force. 


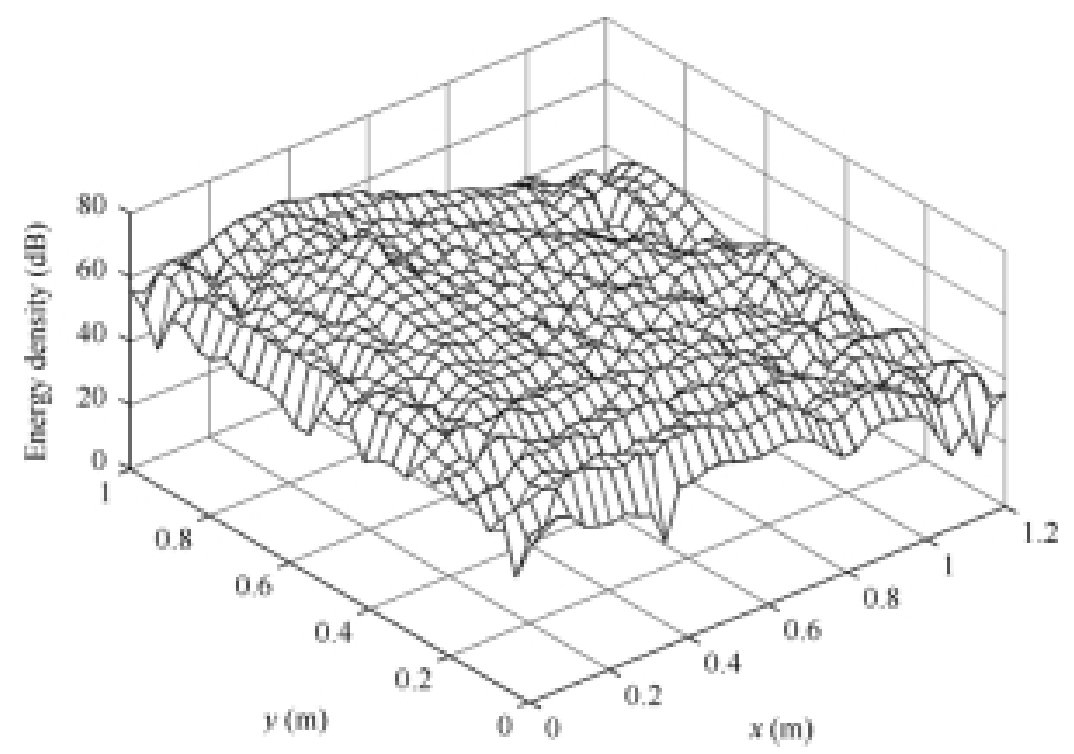

Fig. 23. The classical energy density distribution of the orthotropic plate with $L_{x}=1.2 \mathrm{~m}$ and $L_{y}=1 \mathrm{~m}$ when $x_{o}=0.3 \mathrm{~m}, y_{o}=0.7 \mathrm{~m}$, $f=5 \mathrm{kHz}$ and $\eta=0.1$. The reference energy density is $1 \times 10^{-12} \mathrm{~J} / \mathrm{m}^{2}$.

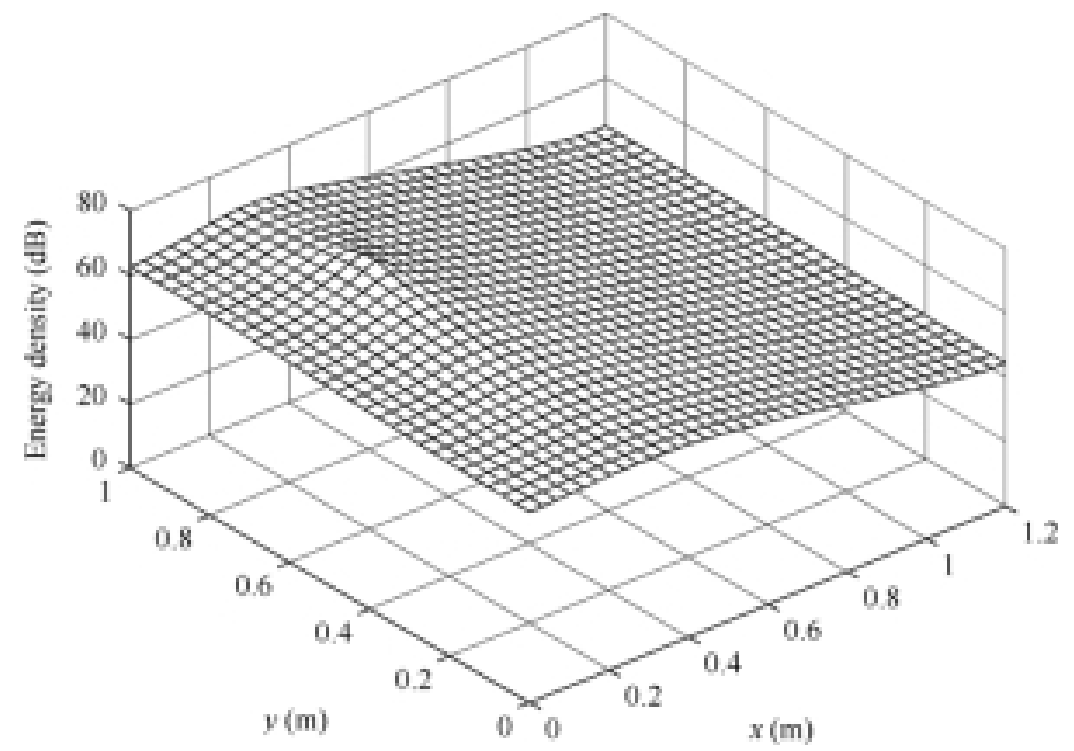

Fig. 24. The approximate energy density distribution of the orthotropic plate with $L_{x}=1.2 \mathrm{~m}$ and $L_{y}=1 \mathrm{~m}$ when $x_{o}=0.3 \mathrm{~m}, y_{o}=0.7 \mathrm{~m}$, $f=5 \mathrm{kHz}$ and $\eta=0.1$. The reference energy density is $1 \times 10^{-12} \mathrm{~J} / \mathrm{m}^{2}$.

The time- and locally space-averaged far-field energy density is calculated by the analytical solution (Eq. (30)) of the energy differential equation developed in this work for a vibrating orthotropic plate. The intensity field in the plate is obtained from Eqs (35) and (36). These energy density distribution and power transmission path predicted by the approximate energy flow model are compared to the energetics obtained from the classical modal solution for the displacement of the corresponding plate (APPENDIX 2). The dimensions and thickness of the plate are $L_{x}=L_{y}=1 \mathrm{~m}$ and $h=1 \mathrm{~mm}$, respectively, and the material properties of the plate are those of aluminum. It is assumed that the plate is stiffened along the $y$-direction and thus $D_{y}$ is 20 times greater than $D_{x}$. The force is located at $x_{o}=0.5 \mathrm{~m}$ and $y_{o}=0.5 \mathrm{~m}$ in the plate. 
In the first example, the exciting frequency is $f=$ $500 \mathrm{~Hz}$ and the damping of the plate is $\eta=0.02$. The energy density distributions of the classical modal displacement solution and the energy flow model solution are shown in Figs 3 and 4, respectively. From the comparison of the results, it is known that the energy flow model solution varies very slowly and well represents a global variation of the classical solution. Figures 5 and 6 show the energy transmission path (intensity field) predicted by classical modal analysis and energy flow models, respectively.

In the next example, the frequency is $f=500 \mathrm{~Hz}$ and the damping of the plate is increased to $\eta=0.2$. The classical and energy flow solutions of the energy density are shown in Figs 7 and 8, respectively. Compared with the previous case seen in Figs 3 and 4, this energy density decreases more quickly due to large damping. For the medium-to-high frequency problems, the averaged values which show the global information can be used meaningfully as observed in Fig. 8. The intensity fields in the plate are shown in Figs 9 and 10, and in comparison with Figs 5 and 6, it is found that as the damping of the plate increases, the intensity field computed from the classical modal solution becomes closer to the EFA intensity field.

When the frequency is $f=5 \mathrm{kHz}$ and the damping loss factor is $\eta=0.02$, the energy density distributions are shown in Figs 11 and 12. The approximate solution well represents the global variation of the classical solution. The approximate energy density decreases slowly in space due to small damping. The intensity distributions are illustrated in Figs 13 and 14. If the damping is increased to $\eta=0.2$ with the same exciting frequency, the classical energy solution is more smoothed and becomes closer to the approximate energy solution as shown in Figs 15 and 16. With high frequency and a large value of damping, the approximate intensity field also becomes closer to the classical intensity field as illustrated in Figs 17 and 18.

Since the orthotropic plate has different bending stiffnesses in two perpendicular directions, its energy distribution differs from that of the isotropic plate. Figures 19 and 20 show the difference between the two energy distribution patterns. While the energy of the isotropic plate flows uniformly in all directions from the source over the entire plate as shown in Fig. 21, the direction of the energy flow in the orthotropic plate depends on how the plate is stiffened or reinforced, as shown in Fig. 22.

Additionally, it can be also found that the approximate energy solution well represents the global vari- ation of the classical solution when the side lengths of the plate are unequal and the exciting force is not centrally located. Figures 23 and 24 show the corresponding distributions of the classical and approximate energy density, respectively, where the dimensions of the plate are $L_{x}=1.2 \mathrm{~m}$ and $L_{y}=1 \mathrm{~m}$ and the force is located at $x_{o}=0.3 \mathrm{~m}$ and $y_{o}=0.7 \mathrm{~m}$ in the plate. The exciting frequency is $f=5 \mathrm{kHz}$ and the damping is $\eta=0.1$.

\section{Conclusions}

In this work, the energy flow model for transversely vibrating orthotropic plates has been newly developed in order to extend the application range of the Energy Flow Analysis (EFA) method to the medium-to-high frequency vibration of orthotropic plate structures. To derive the corresponding energy differential equation, the far-field components of the plane wave were used and time- and space-averaging were performed over a period and a half wavelength with the assumption that the damping is small. The developed energy flow model has a more general form covering the differences in the bending stiffness, and the EFA model of the isotropic plate can be easily derived as a specific case of the developed model. From numerical examples, the approximate energy and intensity field obtained by the derived energy equation are seen to well represent the global variation of the response with the reliable results.

Further studies are recommended on the development of new energy flow models for in-plane waves of the orthotropic plate and on the prediction of the energy and intensity field in coupled orthotropic plates.

\section{References}

[1] D.J. Nefske and S.H. Sung, Power flow finite element analysis of dynamic systems: basic theory and application to beams, Journal of Vibration, Acoustics, Stress and Reliability in Design 111 1989, 94-100.

[2] D.U. Noiseux, Measurement of Power Flow in Uniform Beams and Plates, Journal of the Acoustical Society of America 47(1) (1970), 238-247.

[3] J.C. Wohlever and R.J. Bernhard, Mechanical Energy Flow Models of Rods and Beams, J. Sound Vib. 153(1) (1992), 1-19.

[4] L. Cremer, M. Heckl and E.E. Ungar, Structure-Borne Sound, Springer-Verlag, Berlin, 1973.

[5] M.S. Troitsky, Stiffened Plates; bending, stability and vibrations, Elsevier, Amsterdam, 1976.

[6] O.M. Bouthier and R.J. Bernhard, Models of Space-Averaged Energetics of Plates, AIAA J. 30(3) (1992), 616-623. 
[7] O.M. Bouthier and R.J. Bernhard, Simple Models of the Energetics of Transversely Vibrating Plates, J. Sound Vib. 182(1) (1995), 149-164.

[8] O.M. Bouthier, R.J. Bernhard and C. Wohlever, Energy and Structural Intensity Formulations of Beam and Plate Vibrations, 3rd International Congress on Intensity Techniques, 1990, pp. 37-44.

[9] P.E. Cho, Energy Flow Analysis of Coupled Structures, Ph.D. Dissertation, Purdue University, 1993.

[10] R. Szilard, Theory and Analysis of Plates; classical and numerical methods, Prentice-Hall, Englewood Cliffs, 1974.

[11] S.A. Ambartsumyan, Theory of Anisotropic Plates; strength, stability and vibrations, Hemisphere Pub., New York, 1991.

[12] S. Timoshenko and W. Woinowski-Krieger, Theory of Plates and Shells, (2nd ed.), McGraw-Hill, New York, 1959.

[13] V.D. Belov, S.A. Rybak and B.D. Tartakovskii, Propagation of Vibrational Energy in Absorbing Structures, J. Soviet Physics Acoustics 23(2) (1977), 115-119.

\section{Appendix 1. Space-average and omission of higher order terms of damping loss factor}

If Eq. (16) is expanded, the time-averaged total energy density can be rewritten as

$$
<e>=<e_{1}>+<e_{2}>\text {, }
$$

where $\left\langle e_{1}>\right.$ and $<e_{2}>$ are defined here by

$$
\begin{aligned}
<e_{1}>= & \frac{1}{4} \operatorname{Re}\left\{\left(D_{x c}\left|k_{x}\right|^{4}\right.\right. \\
& +2 \sqrt{\nu_{x} \nu_{y}} \sqrt{D_{x c} D_{y c}} k_{x}^{2}\left(k_{y}^{2}\right)^{*} \\
& +2\left(1-\sqrt{\nu_{x} \nu_{y}}\right) \sqrt{D_{x c} D_{y c}}\left|k_{x}\right|^{2}\left|k_{y}\right|^{2} \\
& \left.+D_{y c}\left|k_{y}\right|^{4}+m \omega^{2}\right) \\
& \times\left(\left|[A]^{--}\right|^{2}+\left|[B]^{+-}\right|^{2}\right. \\
& \left.\left.+\left|[C]^{-+}\right|^{2}+\left.|[D]|^{++}\right|^{2}\right)\right\}
\end{aligned}
$$

and

$$
\begin{aligned}
& <e_{2}> \\
= & \frac{1}{4} \operatorname{Re}\left\{\left(D_{x c}\left|k_{x}\right|^{4}\right.\right. \\
& +2 \sqrt{\nu_{x} \nu_{y}} \sqrt{D_{x c} D_{y c}} k_{x}^{2}\left(k_{y}^{2}\right)^{*} \\
& +2\left(1-\sqrt{\nu_{x} \nu_{y}}\right) \sqrt{D_{x c} D_{y c}}\left|k_{x}\right|^{2}\left|k_{y}\right|^{2} \\
& \left.+D_{y c}\left|k_{y}\right|^{4}+m \omega^{2}\right) \times\left([A]^{--}\left([B]^{+-}\right)^{*}\right. \\
& +[B]^{+-}\left([A]^{--}\right)^{*}+[A]^{--}\left([C]^{-+}\right)^{*} \\
& +[C]^{-+}\left([A]^{--}\right)^{*}+[A]^{--}\left([D]^{++}\right)^{*} \\
& +[D]^{++}\left([A]^{--}\right)^{*}+[B]^{+-}\left([C]^{-+}\right)^{*} \\
& +[C]^{-+}\left([B]^{+-}\right)^{*}+[B]^{+-}\left([D]^{++}\right)^{*}
\end{aligned}
$$

$$
\begin{aligned}
& +[D]^{++}\left([B]^{+-}\right)^{*}+[C]^{-+}\left([D]^{++}\right)^{*} \\
& \left.+[D]^{++}\left([C]^{-+}\right)^{*}\right) \\
& -2\left(1-\sqrt{\nu_{x} \nu_{y}}\right) \sqrt{D_{x c} D_{y c}}\left|k_{x}\right|^{2}\left|k_{y}\right|^{2} \\
& \left([A]^{--}\left([B]^{+-}\right)^{*}+[B]^{+-}\left([A]^{--}\right)^{*}\right. \\
& +[A]^{--}\left([C]^{-+}\right)^{*}+[C]^{-+}\left([A]^{--}\right)^{*} \\
& -[A]^{--}\left([D]^{++}\right)^{*}-[D]^{++}\left([A]^{--}\right)^{*} \\
& -[B]^{+-}\left([C]^{-+}\right)^{*}-[C]^{-+}\left([B]^{+-}\right)^{*} \\
& +[D]^{++}\left([B]^{+-}\right)^{*}+[C]^{-+}\left([D]^{++}\right)^{*} \\
& \left.+[D]^{++}\left([C]^{-+}\right)^{*}\right) .
\end{aligned}
$$

Substituting the first above equation into Eq. (19) yields the time- and locally space-averaged total energy density:

$$
\begin{aligned}
<\tilde{e}>= & \frac{k_{x l} k_{y l}}{\pi^{2}} \int_{0}^{\pi / k_{y l}} \int_{0}^{\pi / k_{x l}}<e_{1}> \\
& +<e_{2}>d x d y \\
= & <\tilde{e}_{1}>+<\tilde{e}_{2}>.
\end{aligned}
$$

Then, if the damping of the plate is small, the exponential function in the right-hand side can be assumed to be nearly constant on the intervals of integration $0 \leqslant x \leqslant\left(\pi / k_{x l}\right)$ and $0 \leqslant y \leqslant\left(\pi / k_{y l}\right)$, the integrals for the first unknown term $\left|[A]^{--}\right|^{2}$ become:

$$
\begin{aligned}
& \int_{0}^{\pi / k_{y l}} \int_{0}^{\pi / k_{x l}}|A|^{2} e^{-(\eta / 2) k_{s l} x-(\eta / 2) k_{y l} y} d x d y \\
& \approx|A|^{2} e^{--} \int_{0}^{\pi / k_{y l}} \int_{0}^{\pi / k_{x l}} d x d y \\
& =\frac{\pi^{2}}{k_{x l} k_{y l}}|A|^{2} e^{--} .
\end{aligned}
$$

Through the same process, $<\tilde{e}_{1}>$ can be obtained by

$$
\begin{aligned}
& <\tilde{e}_{1}>= \\
& \frac{1}{4} \operatorname{Re}\left\{D_{x c}\left|k_{x}\right|^{4}+2 \sqrt{\nu_{x} \nu_{y}} \sqrt{D_{x c} D_{y c}} k_{x}^{2}\left(k_{y}^{2}\right)^{*}\right. \\
& +2\left(1-\sqrt{\nu_{x} \nu_{y}}\right) \sqrt{D_{x c} D_{y c}}\left|k_{x}\right|^{2}\left|k_{y}\right|^{2} \\
& \left.+D_{y c}\left|k_{y}\right|^{4}+m \omega^{2}\right\} \\
& \times\left(|A|^{2} e^{--}+|B|^{2} e^{+-}+|C|^{2} e^{-+}+|D|^{2} e^{++}\right) .
\end{aligned}
$$

The integrals for the unknown term $[A]^{--}\left([B]^{+-}\right)^{*}$ can be obtained in the similar way: 


$$
\begin{aligned}
& \int_{0}^{\pi / k_{y l}} \int_{0}^{\pi / k_{x l}}[A]^{--}\left([B]^{+-}\right)^{*} d x d y \\
= & A B^{*} \int_{0}^{\pi / k_{y l}} e^{-(\eta / 2) k_{y l} y} \\
& \int_{0}^{\pi / k_{x l}} e^{-2 j k_{x l} x} d x d y \\
= & A B^{*} \int_{0}^{\pi / k_{y l}} e^{-(\eta / 2) k_{y l} y} \\
& \left.\frac{e^{-2 j k_{x l} x}}{-2 j k_{x l}}\right|_{x=0} ^{x=\pi / k_{x l}} d y \\
= & 0 .
\end{aligned}
$$

As seen in the above equation, $<\tilde{e}_{2}>$ is nullified, and the time- and locally space-averaged total energy density $<\tilde{e}>$ comes to be equal to $<\tilde{e}_{1}>$.

When the coefficient terms, $\operatorname{Re}\{\ldots\}$, are considered, they include high order terms of damping loss factor $\eta$ as follows:

$$
\begin{aligned}
& \operatorname{Re}\left\{D_{x c}\left|k_{x}\right|^{4}+2 \sqrt{\nu_{x} \nu_{y}} \sqrt{D_{x c} D_{y c}} k_{x}^{2}\left(k_{y}^{2}\right)^{*}\right. \\
& +2\left(1-\sqrt{\nu_{x} \nu_{y}}\right) \sqrt{D_{x c} D_{y c}}\left|k_{x}\right|^{2}\left|k_{y}\right|^{2} \\
& \left.+D_{y c}\left|k_{y}\right|^{4}+m \omega^{2}\right\} \\
= & \left|1-j \frac{\eta}{4}\right|^{4}\left(D_{x} k_{x l}^{4}+2 \sqrt{\nu_{x} \nu_{y}} \sqrt{D_{x} D_{y}} k_{k l}^{2} k_{y l}^{2}\right. \\
& +2\left(1-\sqrt{\nu_{x} \nu_{y}}\right) \sqrt{D_{x} D_{y}} k_{x l}^{2} k_{y l}^{2} \\
& \left.+D_{y} k_{y l}^{4}\right)+m \omega^{2} \\
& \left(1+\frac{\eta^{2}}{8}+\frac{\eta^{4}}{256}\right) \\
& \left(\sqrt{D_{x}} k_{x l}^{2}+\sqrt{D_{y}} k_{y l}^{2}\right)^{2}+m \omega^{2} .
\end{aligned}
$$

Neglecting all of the second and higher order terms of the damping loss factor with the dispersion relation (Eq. (6)), the time- and locally space-averaged total energy density $<\tilde{e}>$ can be finally obtained as the simplified Eq. (18).

Similarly, when spatially averaging the intensity (Eqs (14) and (15)) over a half wavelength, one can obtain the following expressions:

$$
\begin{aligned}
& <\tilde{q}_{x}> \\
= & \frac{\omega}{2} \operatorname{Re}\left\{k_{x}\left(D_{x c} k_{x}^{2}+\sqrt{D_{x c} D_{y c}} k_{y}^{2}\right)\right. \\
& +D_{x c}\left(k_{x}^{2}+\nu_{y} k_{y}^{2}\right) k_{x}^{*} \\
& \left.+\left(1-\sqrt{\nu_{x} \nu_{y}}\right) \sqrt{D_{x c} D_{y c}} k_{x}\left|k_{y}\right|^{2}\right\}
\end{aligned}
$$

$$
\begin{aligned}
& \times\left(|A|^{2} e^{--}-|B|^{2} e^{+-}\right. \\
& \left.+|C|^{2} e^{-+}-|D|^{2} e^{++}\right)
\end{aligned}
$$

and

$$
\begin{aligned}
& <\tilde{q}_{y}> \\
= & \frac{\omega}{2} \operatorname{Re}\left\{k_{y}\left(D_{y c} k_{y}^{2}+\sqrt{D_{x c} D_{y c}} k_{x}^{2}\right)\right. \\
& +D_{y c}\left(k_{y}^{2}+\nu_{x} k_{x}^{2}\right) k_{y}^{*} \\
& \left.+\left(1-\sqrt{\nu_{x} \nu_{y}}\right) \sqrt{D_{x c} D_{y c}} k_{y}\left|k_{x}\right|^{2}\right\} \\
& \times\left(|A|^{2} e^{--}+|B|^{2} e^{+-}\right. \\
& \left.-|C|^{2} e^{-+}-|D|^{2} e^{++}\right) .
\end{aligned}
$$

The coefficient terms in the above equations have high order terms of damping loss factor that is assumed to be small:

$$
\begin{aligned}
& \operatorname{Re}\left\{k_{x}\left(D_{x c} k_{x}^{2}+\sqrt{D_{x c} D_{y c}} k_{y}^{2}\right)\right. \\
& +D_{x c}\left(k_{x}^{2}+\nu_{y} k_{y}^{2}\right) k_{x}^{*} \\
& \left.+\left(1-\sqrt{\nu_{x} \nu_{y}}\right) \sqrt{D_{x c} D_{y c}} k_{x}\left|k_{y}\right|^{2}\right\} \\
& =\left(1-\frac{\eta^{2}}{16}+\frac{\eta^{4}}{64}\right) k_{x l}\left(D_{x} k_{x l}^{2}+\sqrt{D_{x} D_{y}} k_{y l}^{2}\right) \\
& +\left(1+\frac{7}{16} \eta^{2}-\frac{\eta^{4}}{64}\right) D_{x}\left(k_{x l}^{2}+\nu_{y} k_{y l}^{2}\right) k_{x l} \\
& +\left(1+\frac{5}{16} \eta^{2}+\frac{\eta^{4}}{64}\right) k_{x l}\left(1-\sqrt{\nu_{x} \nu_{y}}\right) \\
& \sqrt{D_{x} D_{y}} k_{y l}^{2} .
\end{aligned}
$$

and

$$
\begin{aligned}
& \operatorname{Re}\left\{k_{y}\left(D_{y c} k_{y}^{2}+\sqrt{D_{x c} D_{y c}} k_{x}^{2}\right)\right. \\
& +D_{y c}\left(k_{y}^{2}+\nu_{x} k_{x}^{2}\right) k_{y}^{*} \\
& \left.+\left(1-\sqrt{\nu_{x} \nu_{y}}\right) \sqrt{D_{x c} D_{y c}} k_{y}\left|k_{x}\right|^{2}\right\} \\
& =\left(1-\frac{\eta^{2}}{16}+\frac{\eta^{4}}{64}\right) k_{y l}\left(D_{y} k_{y l}^{2}+\sqrt{D_{x} D_{y}} k_{x l}^{2}\right) \\
& +\left(1+\frac{7}{16} \eta^{2}-\frac{\eta^{4}}{64}\right) D_{y}\left(k_{y l}^{2}+\nu_{x} k_{x l}^{2}\right) k_{y l} \\
& +\left(1+\frac{5}{16} \eta^{2}+\frac{\eta^{4}}{64}\right) k_{y l}\left(1-\sqrt{\nu_{x} \nu_{y}}\right) \\
& \sqrt{D_{x} D_{y}} k_{x l}^{2},
\end{aligned}
$$

respectively. Here, neglecting all of the second and higher order terms of the damping loss factor and using the dispersion relation (Eq. (6)) and Betti's reciprocity 
$\left(\nu_{x} D_{y}=\nu_{y} D_{x}\right)$, the time- and locally space-averaged intensity components can be finally obtained as the simplified Eqs (19) and (20).

\section{Appendix 2. Classical modal solution}

The equation of motion for an orthotropic plate excited by a harmonic point force may be given by

$$
\begin{aligned}
& D_{x c} \frac{\partial^{4} w}{\partial x^{4}}+2 \sqrt{D_{x c} D_{y c}} \frac{\partial^{4} w}{\partial x^{2} \partial y^{2}} \\
& +D_{y c} \frac{\partial^{4} w}{\partial y^{4}}+m \frac{\partial^{2} w}{\partial t^{2}} \\
= & F \delta\left(x-x_{o}\right) \delta\left(y-y_{o}\right) e^{j \omega t} .
\end{aligned}
$$

When the plate is simply supported along its edges, the displacement solution of the equation of motion can be expressed by the double sine series of the spatial variables $x$ and $y$ :

$$
\begin{aligned}
w(x, y, t)= & \sum_{m=1}^{\infty} \sum_{n=1}^{\infty} W_{m n} \\
& \sin \left(\frac{m \pi}{L_{x}} x\right) \sin \left(\frac{n \pi}{L_{y}} y\right) \exp (j \omega t) .
\end{aligned}
$$

The force is also expressed by the double series of the sine function in the form:

$$
\begin{aligned}
& F \delta\left(x-x_{o}\right) \delta\left(y-y_{o}\right) \exp (j \omega t) \\
= & \sum_{m=1}^{\infty} \sum_{n=1}^{\infty} F_{m n} \sin \left(\frac{m \pi}{L_{x}} x\right) \\
& \sin \left(\frac{n \pi}{L_{y}} y\right) \exp (j \omega t)
\end{aligned}
$$

Substituting the displacement and force represented by the double series into the above equation of motion yields

$$
\begin{aligned}
& W_{m n} \\
= & {\left[\frac{4}{L_{x} L_{y}} F \sin \left(\frac{m \pi}{L_{x}} x_{o}\right) \sin \left(\frac{n \pi}{L_{y}} y_{o}\right)\right] / } \\
& {\left[D_{x c}\left(\frac{m \pi}{L_{x}}\right)^{4}+2 \sqrt{D_{x c} D_{y c}}\left(\frac{m \pi}{L_{x}}\right)^{2}\right.} \\
& \left.\left(\frac{n \pi}{L_{y}}\right)^{2}+D_{y c}\left(\frac{n \pi}{L_{y}}\right)^{4}-\omega^{2} m\right] .
\end{aligned}
$$



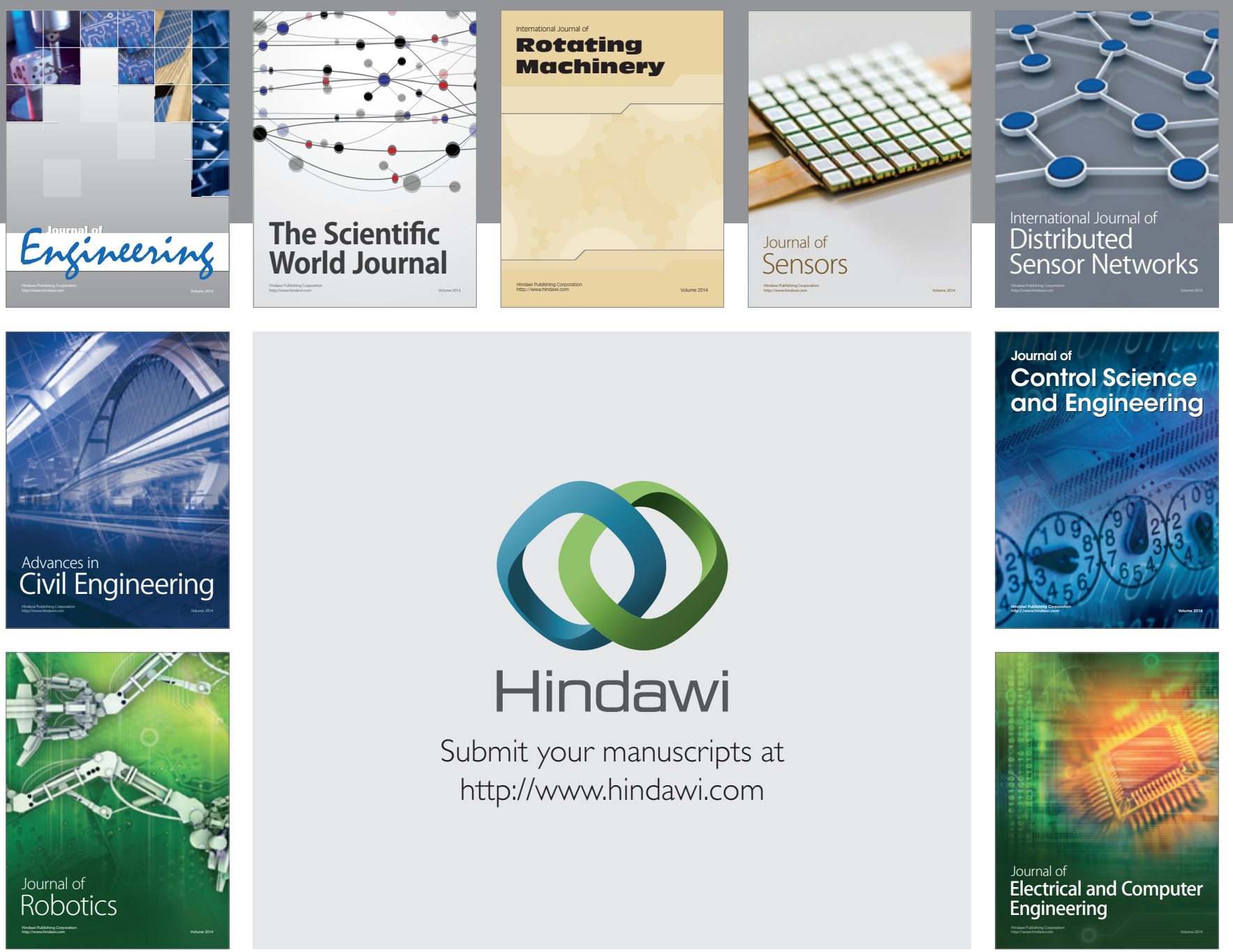

Submit your manuscripts at

http://www.hindawi.com
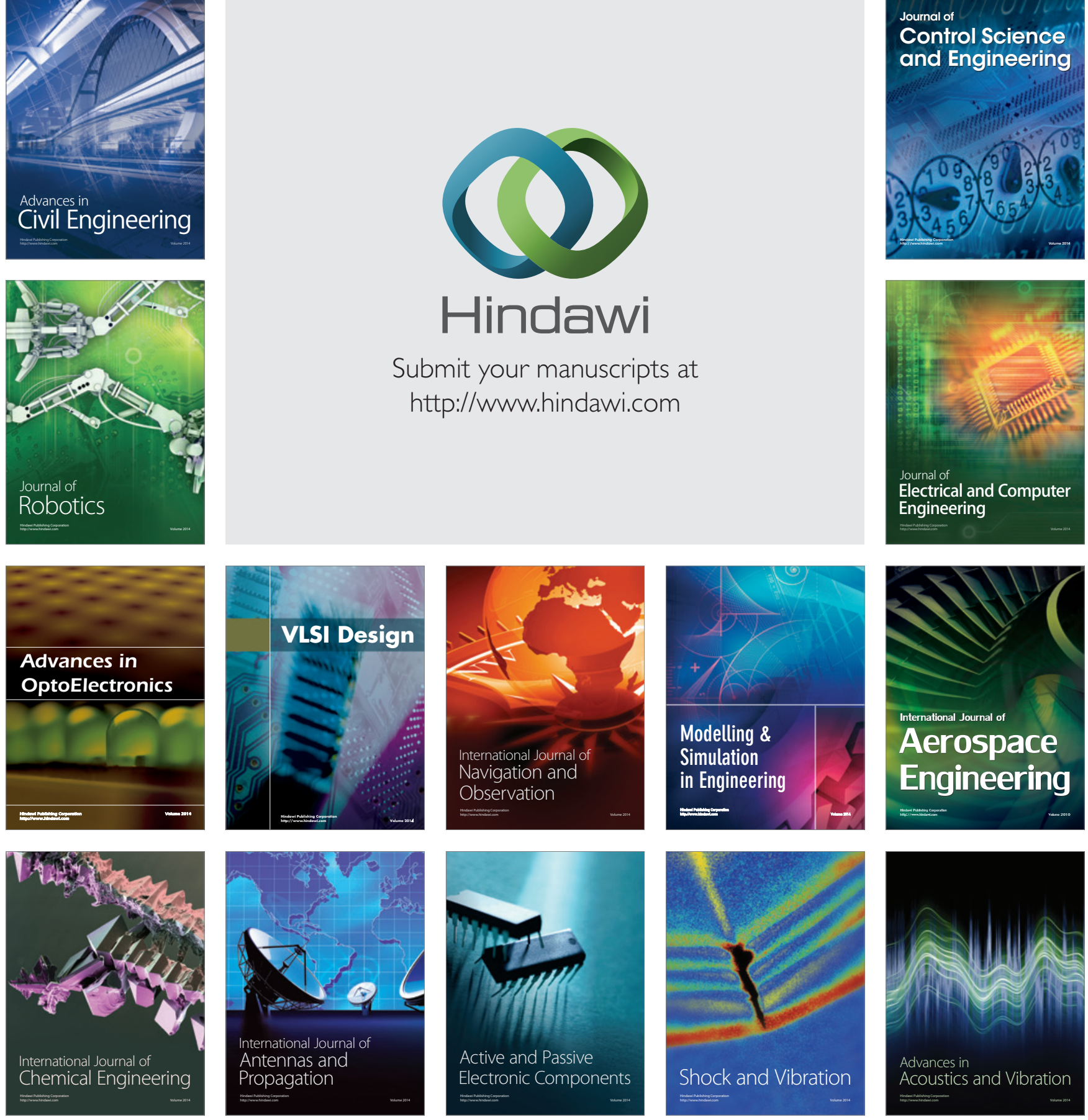